\title{
Male sex hormones increase excitatory neuron production in developing human neocortex
}

\author{
Iva Kelava $^{1 *}$, Ilaria Chiaradia ${ }^{1}$, Laura Pellegrini ${ }^{1}$, Alex T. Kalinka ${ }^{2}$ and Madeline A. Lancaster ${ }^{1 *}$ \\ ${ }^{1}$ MRC Laboratory of Molecular Biology, Francis Crick Avenue, Cambridge, CB2 0QH, UK. \\ ${ }^{2}$ Milner Therapeutics Centre, University of Cambridge, Cambridge Biomedical Campus, Puddicombe Way, Cambridge, CB2 0AW, UK. \\ *Correspondence to: MA Lancaster madeline.lancaster@mrc-lmb.cam.ac.uk, I Kelava ikelava@mrc-lmb.cam.ac.uk
}

\begin{abstract}
The presence of male-female brain differences has long been a controversial topic. Yet simply negating the existence of biological differences has detrimental consequences for all sexes and genders, particularly for the development of accurate diagnostic tools, effective drugs and understanding of disease. The most well-established morphological difference is size, with males having on average a larger brain than females; yet a mechanistic understanding of how this difference arises remains to be elucidated. Here, we use brain organoids to test the roles of sex chromosomes and sex steroids during development. While we show no observable differences between XX and XY brain organoids, sex steroids, namely androgens, increase proliferation of cortical neural progenitors. Transcriptomic analysis reveals effects on chromatin remodelling and HDAC activity, both of which are also implicated in the male-biased conditions autism spectrum disorder and schizophrenia. Finally, we show that higher numbers of progenitors result specifically in increased upper-layer excitatory neurons. These findings uncover a hitherto unknown role for male sex hormones in regulating excitatory neuron number within the human neocortex and represent a first step towards understanding the origin of human sex-related brain differences.
\end{abstract}

Keywords: Sex steroids, androgens, neurogenesis, brain development, autism spectrum disorder

\section{Introduction}

While sex-based brain differences are controversial, one of the most striking and well-accepted differences between males and females is their divergent susceptibility to certain neurological disorders ${ }^{1,2}$, for example, autism spectrum disorder (ASD) ${ }^{3}$ and schizophrenia ${ }^{4-6}$. This differential susceptibility points to fundamental differences in brain structure and/or function. However, the presence of clear, sexspecific neurological characteristics has remained a topic of heated debate, particularly since adult sex-based differences are not dimorphic, but rather a continuum with substantial overlap among individuals ${ }^{7}$. Nonetheless, certain quantitative differences in size are well established, with adult males having on average larger intracranial and total brain volumes and increased grey matter in several brain regions ${ }^{7,8}$. The neonatal presence of size differences, even after correcting for birth weight ${ }^{9,10}$, speaks to a developmental origin. Yet, how these size differences arise, and whether they relate to sex-biased susceptibility to neurological conditions, remain to be determined.

Sexual differentiation has two main sources: the cell-intrinsic chromosomal complement and circulating sex hormones ${ }^{11}$. The effects of sex hormones on brain development have been studied more extensively in mice ${ }^{12}$, where testosterone secreted by the embryonic testes was shown to have its masculinising effects through its conversion to estradiol (the predominant form of estrogens). Thus, counter intuitively, it is estradiol, and not testosterone, which masculinises the mouse brain ${ }^{13}$. However, the binary nature of rodent brain sexualisation and behaviour is strikingly different from humans, and thus, whether such a mechanism is at play in humans is unknown. Furthermore, studies of the cell-biological effects of sex hormones on embryonic neural progenitors $^{14,15}$ have, so far, not been able to provide a mechanistic understanding of size differences, particularly in the human context.

\section{Results}

Androgens increase the number of basal progenitors in developing human neocortex

To gain an understanding of developmental events that may lead to male-female differences in the human context, we used cerebral organoids $^{16}$, which provide a reliable model of early human brain development ${ }^{17}$. We compared male and female organoids, assessed neural progenitor responses to different sex hormones, and analysed the behaviour of cells and their progeny over time. Overall, we saw no differences between organoids made from female (XX) and male (XY) cell lines in various assays (see Methods), suggesting intrinsic sex chromosomal makeup does not influence cortical development, at least in this model system (Fig. 1, Extended Data Fig. 1-2).

Next, to examine the effects of sex steroids, we exposed brain organoids to male sex hormones testosterone (T) and dihydrotestosterone (DHT), and the female hormone estradiol (E2) (Fig. 1a, b). DHT is a more potent androgen and cannot be aromatised into E2, as opposed to $\mathrm{T}^{18}$. Treatments were started at day 17 of the protocol as the organoids at this stage morphologically resemble the time point at which the testosterone surge begins in the human embryo (around 8 gestational weeks $(\mathrm{GW}))^{19}$.

In both male and female cell lines, the most striking phenotype was seen upon treatment with androgens (DHT or T), which led to an increase in intermediate progenitors, a population of basally located cells that undergo a finite number of divisions to produce neurons (Fig. 1c, d, Extended Data Fig. 1a-b). Notably, this phenotype was not seen upon treatment with E2. Staining for the proliferative marker Ki67 revealed an increased proportion of intermediate progenitors that remained proliferative upon DHT treatment (Fig. 1e, f). In addition, we observed an increase in the number of another basal progenitor type known as basal radial glia ${ }^{20,21}$ in DHT-treated organoids (Fig. 1g, h). Taken together, these findings suggest that androgens increase the 
a

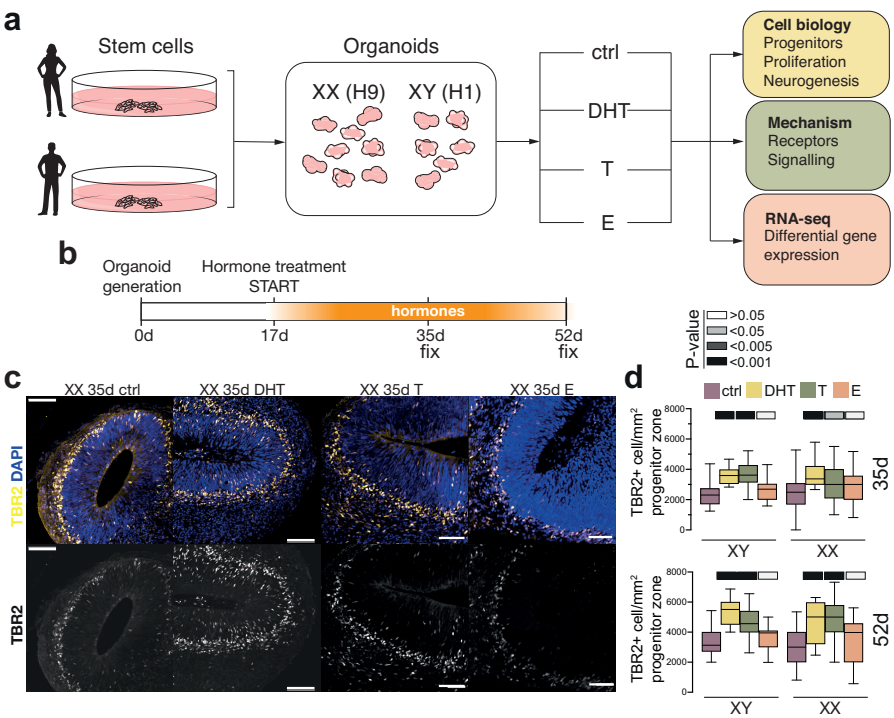

e

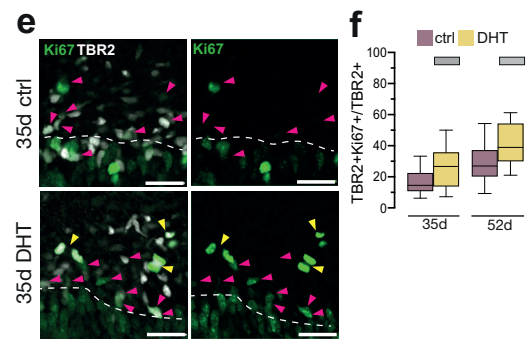

Figure 1. Androgens, but not estrogen, cause an expansion of progenitors

a) Schematic of the project workflow. b) Organoid generation and treatment protocol timeline. c) Female (XX) organoids at 35d, treated with DHT, T and E and stained for the intermediate progenitor marker TBR2 (yellow, white). Note the increase in TBR2+ in DHT/T treated organoids. d) Quantification of TBR2+ cells per $\mathrm{mm}^{2}$ of progenitor layer in male (XY) and female (XX) organoids, at $35 \mathrm{~d}$ and $52 \mathrm{~d}$. e) Image of the ventricular zone/subventricular zone (VZ/SVZ) border in XX 35d organoids stained for Ki67 (green) and TBR2 (white). Cells positive for both Ki67 and TBR2 are indicated with magenta arrowheads. Double positive mitotic cells are indicated with yellow arrowheads. A white dashed line delineates VZ/SVZ border with the ventricular zone below. f) Quantification of Ki67/TBR2 double positive cells, out of all TBR2+ cells, in XX 35d and 52d organoids. g) Quantification of HOPX+ basal radial glia per $\mathrm{mm}^{2} \mathrm{SVZ}$ of XX 52d organoids. h) Immunostaining for HOPX (fire LUT) of XX 52d organoids. Yellow dashed line indicates the ventricular surface. Note the HOPX signal in radial glia. All images are single, $1.2 \mu \mathrm{m}$ optical planes. Scale bars: c) and j) $50 \mu \mathrm{m}, \mathrm{g}) 25 \mu \mathrm{m}$. Whiskers in boxplots are minmax values. Significance values (Mann-Whitney, two tailed) of the treatment, as compared to the control, indicated by different shades of grey.

numbers and proliferation of basal progenitors in the developing human neocortex.

\section{DHT promotes proliferation of apical radial glial stem cells}

To investigate the mechanism of this increase in basal progenitors, we examined the neural stem cells that give rise to basal progenitors and neurons, called radial glia. We observed certain features indicative of radial glial proliferation, namely number of apical mitoses and ventricular length, that appeared increased upon treatment with androgens (Extended Data Fig. 2a, b). We next used viral sparse labelling of the radial glial neural stem cells to perform lineage tracing (Fig. 2a), and observed a significant increase in clone size upon treatment for 8 days with DHT (Fig. 2b, c, e). We then tested whether this effect was specific and reversible by removing hormone and labelling a novel population of radial glia with a different virus (Fig. 2a). After 8 days of no treatment, newly-labeled clone sizes of control and previously-DHT treated organoids were comparable (Fig. 2d, e). These results indicate that androgen signalling acts specifically and reversibly to promote symmetric, proliferative divisions of radial glial stem cells, increasing their output.
Androgens signal through the androgen receptor expressed by radial glial stem cells

Androgen receptor (AR), the canonical androgen signalling mediator, is a nuclear receptor which, upon ligand binding, homodimerizes and translocates to the nucleus, where it acts as a transcription factor ${ }^{22}$. $\mathrm{AR}$ is present in the developing primate cortex ${ }^{23}$, but it is still unclear in which cells of the developing human neocortex. We performed RNAScope $^{24}$ for AR mRNA, which revealed widespread signal in radial glia (Fig. 3a), with a distribution that varied depending on the stage of the cell cycle (Extended Data Fig. 3a, b). Western blot for AR protein revealed a decline over time, whereas organoids exposed to DHT showed a sustained expression of AR protein (Extended Data Fig. 3c). This is in accordance with similar observations in a neural progenitor cell line ${ }^{15}$. Together, these findings indicate that radial glial stem cells are receptive to androgens, and that androgens in turn positively feedback to stabilise AR protein ${ }^{25}$.

To test whether androgens act through AR within radial glia, we used a constitutively active form of AR, AR- $7^{26}$, which enters the nucleus and exerts its function, even in the absence of androgens. GFP tagged AR-V7 showed nuclear distribution, indicating its successful entry into the nucleus (Fig. 3b). Most of these GFP-positive nuclei resided in the apical ventricular zone at 2 days post-electroporation, indicating

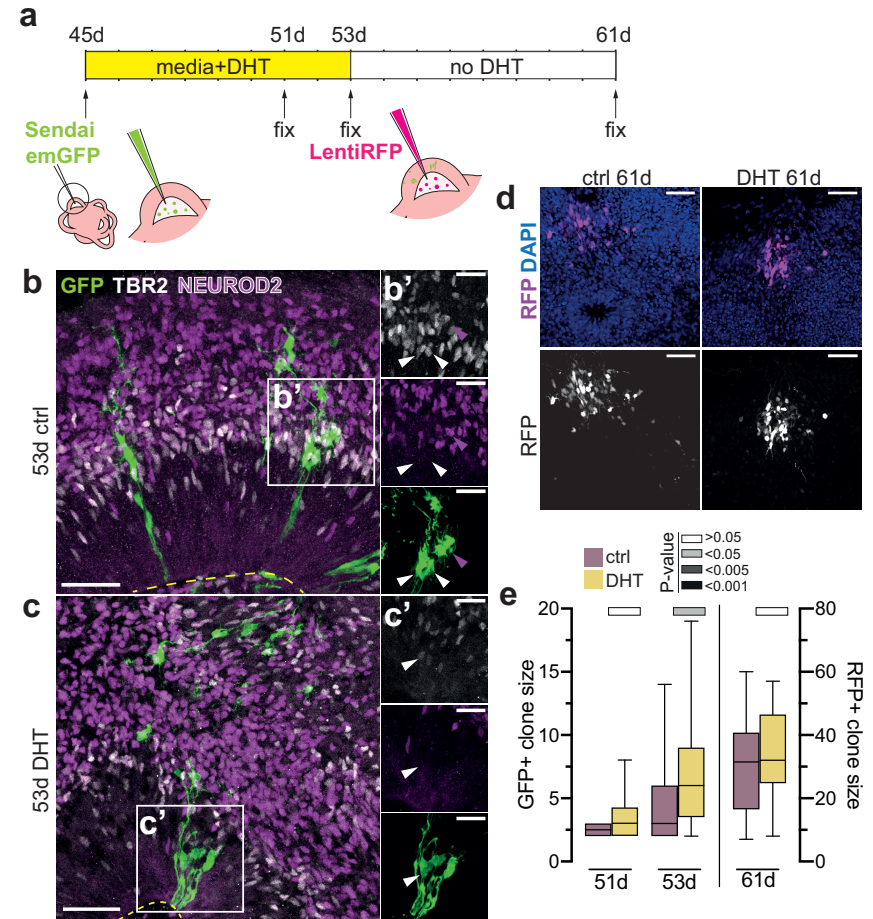

Figure 2. Androgens influence the mode of division of radial glia

a) Timeline and schematic of the Sendai/Lenti virus lineage tracing and analysis, with virus encoding emGFP injected at day 45 and virus encoding RFP injected at day 53. b) XX control organoids, injected with Sendai emGFP at $45 \mathrm{~d}$, fixed at 53d and stained for GFP (green), TBR2 (white) and NEUROD2 (magenta). Yellow dashed line indicates ventricular surface. b') Inset from b). White arrowheads: TBR2/GFP double positive cells. Magenta arrowhead: NEUROD2/GFP double positive cell. Image is a maximum intensity projection of 9 individual $1.2 \mu \mathrm{m}$-thick single optical sections. c) XX DHT-treated organoids, injected with Sendai emGFP at $45 \mathrm{~d}$, fixed at 53d and stained for GFP (green), TBR2 (white) and NEUROD2 (magenta). Yellow dashed line indicates ventricular surface. Image is a maximum intensity projection of 11 individual $1.2 \mu \mathrm{m}$-thick single optical sections. c') Inset from c). White arrowhead: TBR2/GFP double positive cell. Note the distribution of the clone, with most of the GFP+ cell bodies still in the VZ. d) XX organoids, injected with Sendai emGFP at 45d, with Lenti RFP at 53d and fixed after a pulse of DHT treatment or vehicle control. Immunostaining for RFP (magenta, white) Co-stained with DAPI (blue). Images are a maximum projection of 13 (control) and 11 (DHT) individual $1.2 \mu \mathrm{m}$-thick single optical sections. e) Quantification of GFP+ clone size (left side) at $51 \mathrm{~d}$ and $53 \mathrm{~d}$ and RFP+ clone size at $61 \mathrm{~d}$. Individual data points represent individual clones. Scale bars: b), c), d) $50 \mu \mathrm{m}$, b'), c') $25 \mu \mathrm{m}$. Whiskers in boxplots are min-max values Significance values (Mann-Whitney, two tailed) of the treatment, as compared to the control, indicated by different shades of grey. 
a radial glial identity, whereas in control organoids, cells expressing GFP alone were distributed in both apical and more differentiated basal compartments (Fig. 3b). AR-V7-expressing cells were healthy and cycling, as indicated by their Ki67 immunostaining (Fig. 3c), which also revealed a $\sim 60 \%$ increase in proliferation compared with control (Fig. 3d). Along these lines, staining for markers of differentiation revealed a decrease in differentiation of cells expressing AR-V7 (Fig. 3b, e-g).

The AR signalling pathway is most heavily studied in the context of prostate cancer, where downstream signalling can lead to changes in the levels of the transcription factor BRN2 $2^{27}$. BRN2 is present in primate radial glia and is important for neurogenesis ${ }^{28}$. Mirroring the processes described in prostate cancer, we observed a significant reduction in BRN2 in AR-V7-electroporated cells (Fig. 3g, h) further supporting a less differentiated state. Finally, AR-V7 expressing cells also exhibited a significant reduction of the neuronal marker $\mathrm{HuC} / \mathrm{D}$, indicating that they did not become neurons at the same rate as control cells (Fig. 3i).

In order to test the specificity of the effect of expression of AR-V7, we tested expression of a constitutively active form of another sex hormone receptor, estrogen receptor $\alpha(\mathrm{ER} \alpha)^{29,30}$. Expression of a GFP tagged constitutively active ER $\alpha$ Y 537S revealed proliferation status similar to the control at 2 days post-electroporation (Fig. 3c-d), indicating that the effects of AR-V7 were not due to a general response to the constitutive activation of any sex hormone receptor, but were AR specific. We did however observe that at 5 days postelectroporation most of the ER $\alpha$ Y 537S + cells died (Fig. 3d), indicating a later effect on cell survival.

To directly visualise the effects of constitutively active AR, we performed live imaging of organoids electroporated with a control mCherry plasmid and plasmid encoding GFP-tagged AR-V7. As expected, control mCherry-only positive cells divided asymmetrically resulting in one daughter cell remaining a radial glial stem cell, while the other differentiated and left the apical ventricular zone (Extended Data Fig. 4). On the other hand, GFP tagged AR-V7 positive cells divided symmetrically with both daughter cells remaining in the ventricular zone, indicating a radial glial identity (Extended Data Movie 1-3). This behaviour was observed at two different organoid ages, even at 53 days, when most divisions of radial glial stem cells would otherwise be expected to be asymmetric, differentiative divisions.

Together, these findings reveal that when AR signalling is active, radial glial stem cells remain proliferative and increase their numbers. However, our earlier findings of an increased abundance of basal progenitors upon androgen treatment revealed that radial glia in the presence of androgens could still differentiate and produce basal progenitors. These findings at first glance appear contradictory; however, these two experimental approaches accomplished very different things. Whereas the AR-V7 isoform is constitutively active, and therefore would continuously signal in radial glia, application of androgens would only activate AR signalling in those cells that already express AR. Our findings of fluctuating cellular levels of AR (Extended Data Fig. 3b) suggest normally transient responsiveness of these cells, which, during an unresponsive phase, would have a chance to differentiate and generate basal progenitors. AR signalling would therefore be expected to increase the stem cell pool such that upon differentiation, they would produce increased numbers of basal progenitors.
RNA-seq analysis of treated organoids reveals genes involved in androgen response

Androgen induced changes in gene expression in neural progenitors have previously been reported to be subtle $\mathrm{e}^{15}$. Therefore, in order to capture the downstream response with enough transcriptomic coverage to detect such subtle changes we performed RNA-seq on total mRNA isolated from control and androgen-treated organoids (Extended Data Fig. 5a, b). To detect differentially expressed genes, we employed a recently described novel method ${ }^{31}$ designed to ensure high sensitivity while controlling the false discovery rate (see Methods).
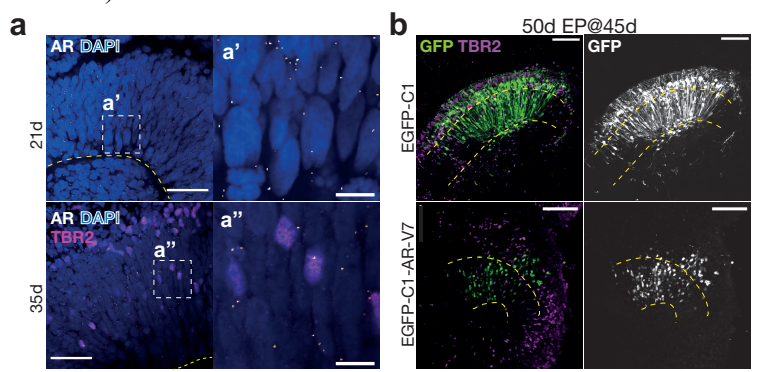

c
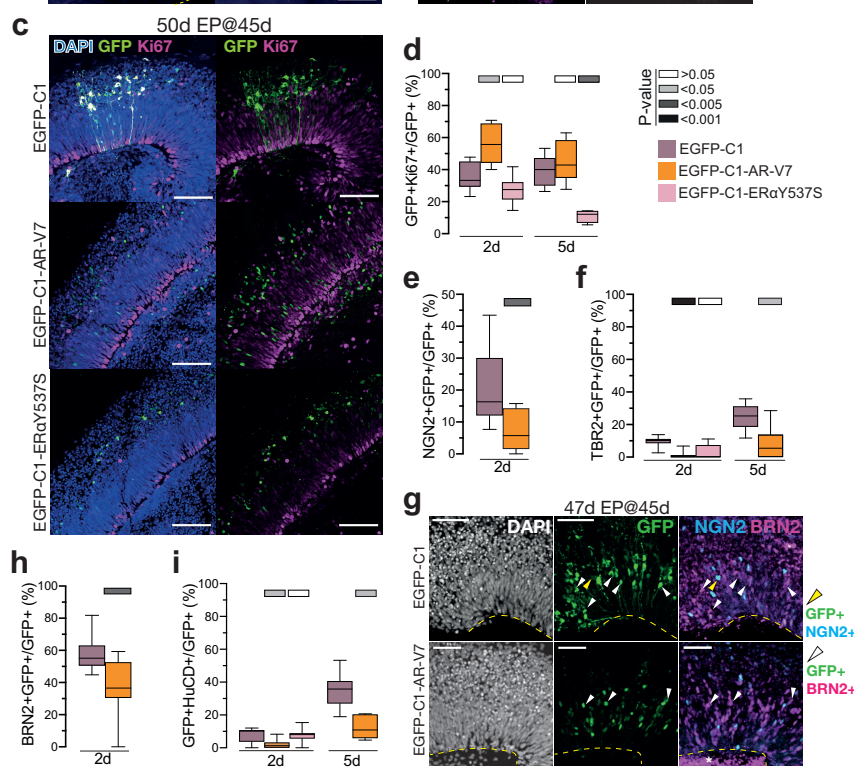

Figure 3. Androgen receptor signalling promotes proliferative identity of radial glia a) RNA Scope for AR of XX 21d (top) and 35d (bottom) organoids. Single AR mRNA puncta (white). Immunostaining for TBR2 (magenta). Co-stained with DAPI (blue). a') Portion of the ventricular zone (VZ) from XX 21d organoid. a") Portion of the VZ from XX 35d organoid. b) Images of XX 35d organoids electroporated at $45 \mathrm{~d}$ and fixed at 50d. EGFP-C1: control plasmid; EGFP-C1-AR-V7: plasmid expressing constitutively active AR. Immunostaining for GFP (green, white) and TBR2 (magenta). Yellow dashed lines demarcate the apical and basal boundaries of the VZ. Note the localization of GFP+ cell bodies in the VZ in EGFP-C1-AR-V7 electroporated organoids. c) Immunostaining for GFP (green) and Ki67 (magenta) on XX 50d organoids, electroporated at 45d. Co-stained with DAPI (blue). EGFP-C1: control plasmid; EGFP-C1-ARV7: plasmid expressing constitutively active AR; EGFP-C1-ER $\alpha$ Y 537S: plasmid expressing constitutively active ER $\alpha$. d) Quantification of the proportion of GFP+ cells costaining for the proliferation marker Ki67 2- and 5 days post electroporation in XX organoids electroporated at $45 \mathrm{~d}$. e) Quantification of the proportion of GFP + cells costaining for the neurogenic marker NGN2 2 days post electroporation in XX organoids electroporated at 45d. f) Quantification of the proportion of GFP+ cells costaining for the intermediate progenitor marker TBR2 2- and 5 days post electroporation in XX organoids electroporated at $45 \mathrm{~d}$. At 5 days post electroporation, most cells electroporated with EGFP-C1-ER $\alpha$ Y537S died. g) XX 47d organoids, electroporated at $45 \mathrm{~d}$. Immunostaining for GFP (green), NGN2 (cyan) and BRN2 (magenta). Co-stained with DAPI (white). Yellow arrowheads: GFP+/NGN2+ cells. White arrowheads: GFP+/BRN2+ cells h) Quantification of the proportion of GFP+ cells costaining for the upper layer neurogenesis marker BRN2 2 days post electroporation in XX organoids electroporated at $45 \mathrm{~d}$. i) Quantification of the proportion of GFP+ cells costaining for the neuronal marker HuC/D 2- and 5 days post electroporation in $\mathrm{XX}$ organoids electroporated at $45 \mathrm{~d}$. At 5 days post electroporation, most cells electroporated with EGFP-C1-ER $\alpha$ Y 537S died. Scale bars: b), c), g) $50 \mu \mathrm{m}$, a) $25 \mu \mathrm{m}$, a'), a") $5 \mu \mathrm{m}$. Whiskers in boxplots are min-max values. Significance values (Mann-Whitney, two tailed) of the treatment, as compared to the control, indicated by different shades of grey. 


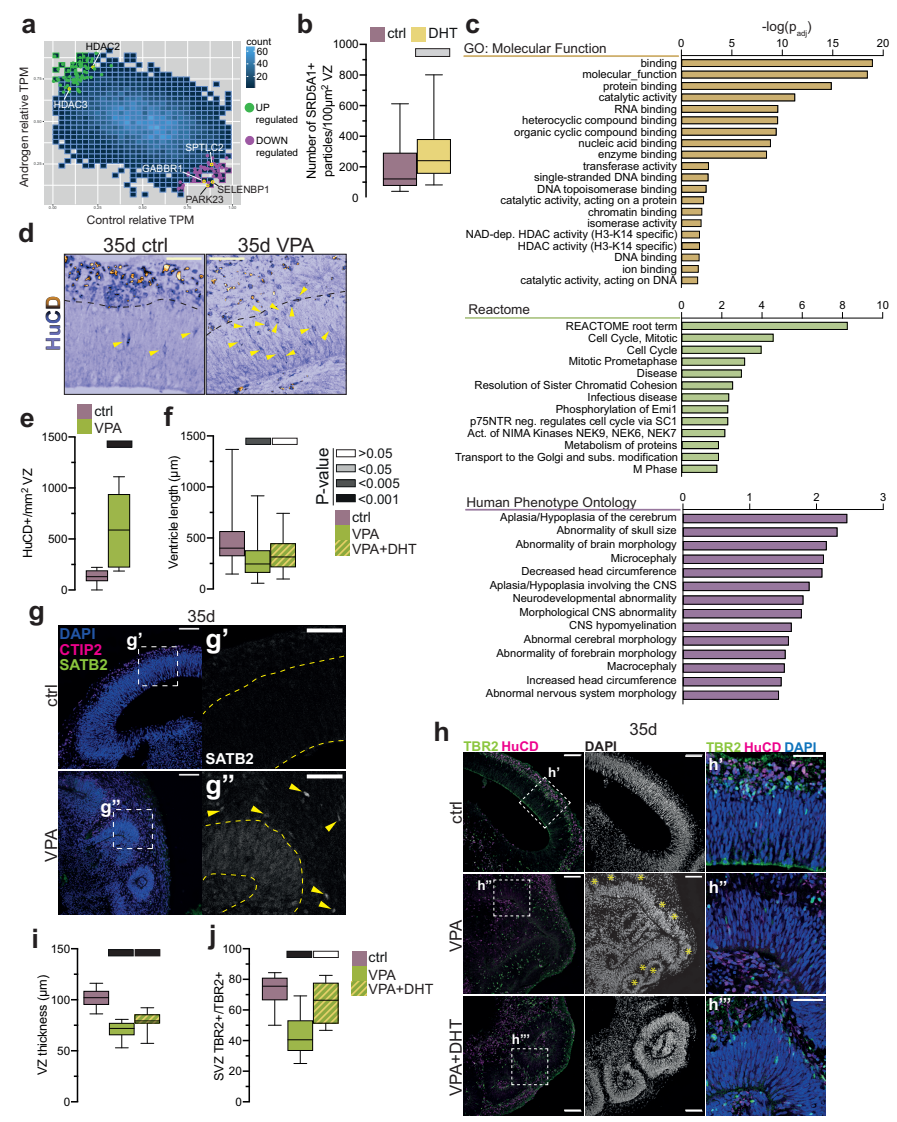

Figure 4. Differential gene expression shows involvement of HDACs in androgen-mediated phenotype

a) A plot showing normalised TPM values of all genes in control and androgen treatment. DEGs were detected using Delboy ${ }^{31}$. Upregulated DEGs are in green. Downregulated DEGs are in magenta. The density of data (count) is indicated by shades of blue. Among the downregulated genes, genes connected with schizophrenia are labelled. b) Quantification of SRD5A1, an upregulated DEG under androgen treatment. SRD5A1 was quantified as the number of SRD5A1+ puncta per $10 \mu \mathrm{m}^{2}$ of the ventricular zone (VZ) in XX 35d control and DHT-treated organoids (see Figure 5f). c) Graph of a number of top significant GO term enrichments in XX $35 \mathrm{~d}$ androgen upregulated genes. d) $\mathrm{HuC} / \mathrm{D}$ immunostaining (purple) in control and VPA-treated XX 35d organoids. e) Quantification of $\mathrm{HuC} / \mathrm{D}+$ cells per $\mathrm{mm}^{2} \mathrm{VZ}$ in control and VPA-treated XX 35d organoids. f) Quantification of ventricular length in $\mu \mathrm{m}$ for XX $35 \mathrm{~d}$ old organoids treated with VPA and VPA+DHT. g) Immunostaining for CTIP2 (magenta), and SATB2 (green, white) on XX 35d control and VPA-treated organoids. Co-stained with DAPI (blue). g'), g") inset from g). g") Note the SATB2+ cells (yellow arrowheads) and SATB2+ staining present in the VZ in VPA-treated organoids. Yellow dashed lines demarcate the apical and basal boundaries of the VZ. h) Immunostaining for TBR2 (green) and $\mathrm{HuC} / \mathrm{D}$ (magenta) in XX 35d organoids, treated with VPA from 17d. Co-stained with DAPI (white, blue). h') h") h"') inserts from h), as indicated. Yellow asterisks indicate neural rosettes forming above the VZ in some VPA-treated organoids. i) Quantification of the thickness of VZ in $\mu \mathrm{m}$ for XX 35d organoids treated with VPA and VPA+DHT. j) Quantification of the percentage of TBR2+ cells in the SVZ, out of all TBR2+ cells, for XX 35d organoids treated with VPA and VPA+DHT. Scale bars: h) $100 \mu \mathrm{m}$, h'), h"), h"'), d), g) $50 \mu \mathrm{m}$. Whiskers in boxplots are min-max values. Significance values (MannWhitney, two tailed) of the treatment, as compared to the control, indicated by different shades of grey.

Comparing control and androgen treated organoids we found 65 upregulated and 51 down-regulated genes (Fig. 4a, Extended Data Table 1). Within the up-regulated genes were several genes with roles in neural development/progenitors: $\mathrm{HDAC}^{32}, \mathrm{HDAC}^{33}, \mathrm{YBX}^{34}$, $\mathrm{EML1}^{35,36}, \mathrm{QARS}^{37}, \mathrm{PLK}^{38}$. Among the upregulated genes, it was encouraging to find $5 \alpha$ reductase (SRD5A1), the enzyme that converts T into DHT. For this gene, we validated the DHT-induced increase on the protein level by quantification of subcellular SRD5A1+ puncta (Fig. 4b).

Two of the upregulated genes, MCM4 and PHB, are listed in the Simons Foundation Autism Research Initiative (SFARI) gene list as strongly associated with ASD, (https://gene.sfari.org/, Category 3 or higher), and several others are involved in $\mathrm{ASD}^{39-41} .10$ of the upregulated and 8 of the downregulated genes also appeared on the list of genes significantly differentially expressed in schizophrenia ${ }^{42}$.
A hypergeometric test of the intersection between the datasets showed that both the overlaps (up- and downregulated genes) were significant $(\mathrm{P}=3.6 \mathrm{E}-4$ and $\mathrm{P}=0.0012$, respectively) (Extended Data Fig. 5c.).

Gene ontology analysis ${ }^{43}$ of downregulated genes showed very low enrichment scores, while upregulated genes were enriched in various terms associated with brain size, developmental disorders, and chromatin activity. In particular, GO: Molecular Function terms were connected to nucleic acid binding/chromatin binding and HDAC activity, Reactome terms were connected to cell cycle and $\mathrm{M}$ phase, and Human Phenotype Ontology terms were connected to neurodevelopmental disorders and CNS abnormality, particularly brain size and head circumference (Fig. 4c).

Interfering with androgen signalling effectors influences differentiation

The effect on HDACs stood out in particular because dysregulated levels of HDACs have been suggested to be involved in masculinisation of the rodent brain $^{44}$ and in pathogenesis of the malebiased disorders $\mathrm{ASD}^{39,45,46}$ and schizophrenia ${ }^{47,48}$, and several genes on the down-regulated list have previously been reported to be associated with schizophrenia (PARK2 $3^{49}$, SELENBP $^{50}$, GABBR $^{51}$, SPTLC $2^{52}$ ).

A potent inhibitor of HDACs, valproic $\operatorname{acid}^{53}$ (VPA), is commonly used as an antiepileptic, but prenatal exposure can lead to malformations of the embryo ${ }^{54}$. In utero exposure to VPA has been associated with increased risk of developing $\mathrm{ASD}^{55}$, potentially by its interference with the normal balance of proliferation and differentiation of neural progenitors ${ }^{56}$. We therefore tested for a functional interaction between HDACs and androgens by treating organoids with VPA. We added VPA at $0.7 \mathrm{mM}$, which is close to therapeutic doses of $\mathrm{VPA}^{57}$. VPA-treated organoids had smaller ventricles and an increased number of neurons in the apical ventricular zone upon VPA treatment, as indicated by HuC/D staining (Fig. 4d-f), suggesting that HDAC inhibition promotes depletion of the radial glial stem cell pool and premature neuronal differentiation, and indicating that HDACs, like androgens, normally inhibit differentiation of radial glial stem cells. Furthermore, staining for later-born upper layer neurons revealed a premature production of these neurons upon VPA treatment (Fig. 4g), suggesting a premature switch in neurogenic potential, similar to previously shown HDAC2 $2^{58}$ and $\mathrm{HDAC} 33^{33}$ knock-out studies.

To test whether HDACs acted downstream of androgen signalling, we investigated whether organoids treated with VPA also exhibited phenotypes affecting progenitor populations. In addition to the smaller ventricular length, we observed a noticeably thinner ventricular zone (Fig. $\mathbf{4 h}$, i) and a reduced number of intermediate progenitors (Fig. $\mathbf{4 h}, \mathbf{j}$ ). While administration of DHT together with VPA did not rescue the thickness of the ventricular zone in VPAtreated organoids (Fig. 4i), it did rescue the reduced ventricular length and returned intermediate progenitor distribution to control levels (Fig. $4 \mathbf{h}, \mathbf{j})$. This ability of DHT to rescue aspects of the VPA-induced phenotype, namely those processes also affected by androgen signalling, suggests that HDAC activity functions downstream of androgen signalling in cortical neurogenesis.

\section{Neural progenitors of the ventral forebrain respond to DHT differently}

The finding that a number of the differentially expressed genes were associated with both ASD and schizophrenia points to a role for 


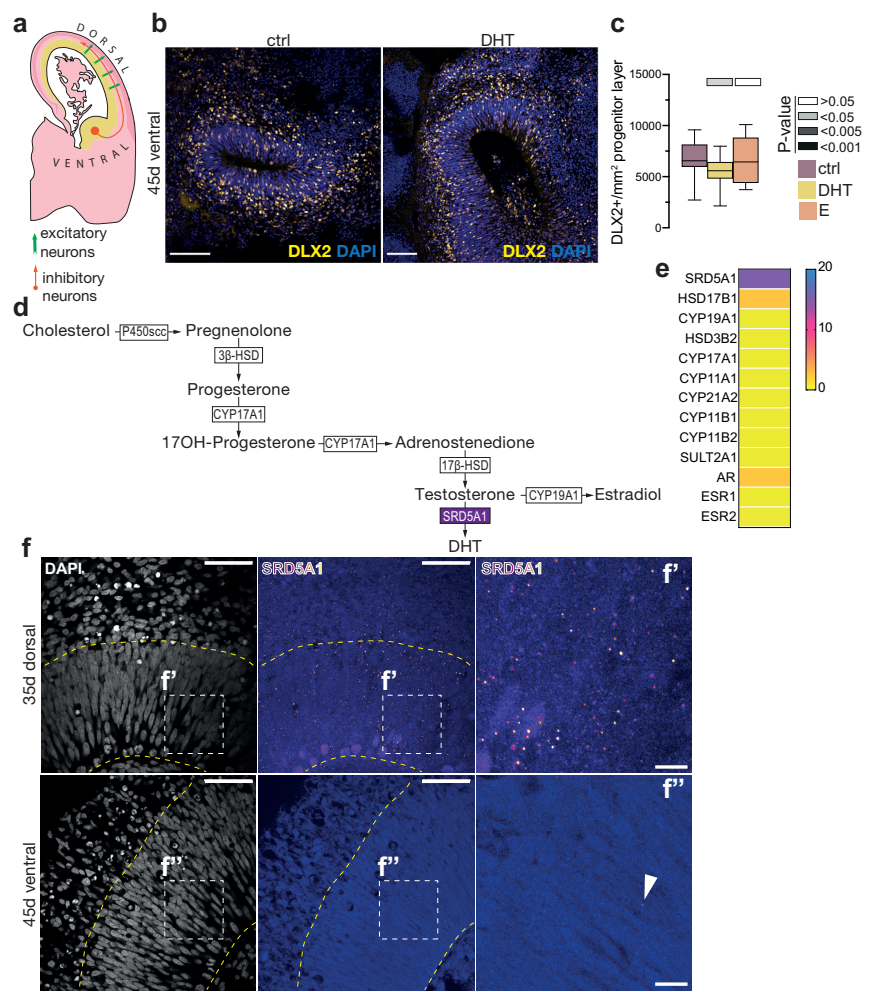

Figure 5. Androgens do not promote expansion of ventral progenitors

a) Schematic of neurogenesis in the human developing brain at $\sim 12 \mathrm{GW}$. Excitatory neurons (green arrows) are born within the dorsal cortex and migrate basally to form the cortical plate. Inhibitory neurons (orange arrow) are born in the ventral telencephalon and migrate towards the dorsal side to incorporate themselves into the cortical plate. Yellow: progenitor zone, dark pink: cortical plate. b) Immunostaining for a ventral intermediate progenitor marker DLX2 (yellow) on XX 45d ventral brain organoids. Co-stained with DAPI (blue). c) Quantification of DLX2+ cells per $\mathrm{mm}^{2}$ progenitor layer for control, DHT and E-treated organoids. d) Schematic of the steroidogenic pathway leading to the formation of DHT and estrogen (estradiol). SRD5A1 is indicated in purple. e) Heatmap of steroidogenic enzyme expression from XX 35d RNA-seq. Values are in tmp. f) Comparison of SRD5A1 (fire LUT) immunostaining on XX dorsal 35d and ventral $45 \mathrm{~d}$ organoids. Co-stained with DAPI (white). Yellow dashed lines demarcate the apical and basal boundaries of the VZ. f') Portion of the VZ in XX dorsal 35d organoids stained for SRD5A1. Note the SRD5A1+ puncta. f') Portion of the VZ in XX ventral 45d organoids stained for SRD5A1. A white arrowhead indicates an SRD5A1+ punctum. Scale bars: f) $\left.\left.50 \mu \mathrm{m}, \mathrm{f}^{\prime}\right), \mathrm{f}^{\prime \prime}\right)$ $10 \mu \mathrm{m}$. Whiskers in boxplots are min-max values. Significance values (Mann-Whitney, two tailed) of the treatment, as compared to the control, indicated by different shades of grey.

androgens in the sex-bias of these conditions ${ }^{47-49}$. There is increasing evidence in both ASD and schizophrenia for an imbalance in excitatory and inhibitory neurons ${ }^{59,60}$. Interestingly, excitatory and inhibitory neurons have distinct developmental origins. While excitatory neurons are produced by radial glial stem cells within the cortex, inhibitory neurons are generated in the ventral forebrain and later migrate into the cerebral cortex from $\operatorname{afar}^{61,62}$ (Fig. 5a). Because of these distant origins, we hypothesised that circulating androgens may impact inhibitory neural progenitors in the ventral telencephalon differently to precursors of excitatory neurons in the dorsal cortex. We therefore treated ventral forebrain organoids with sex steroid hormones and found that DHT actually caused a slight decrease in the number of ventral intermediate progenitors (Fig. 5b, c). This effect was androgen specific since E2 treatment had no effect. This indicates that different parts of the brain respond to androgens in markedly different ways, which could have significant implications for the final neuronal composition of the cortex.

Importantly, the finding that DHT is capable of eliciting these androgen responses in neural progenitors suggests that, because DHT cannot be aromatized, these effects must not be through its conversion to estradiol, unlike in rodents ${ }^{13}$. In support of this conclusion, we found that aromatase (CYP19A1), the enzyme that converts testosterone into estrogen ${ }^{18}$ (Fig. 5d), was absent in our RNA-seq dataset (Fig. 5e), whereas SRD5A1 ${ }^{18}$, the enzyme that locally converts $\mathrm{T}$ to $\mathrm{DHT}^{63}$, was abundantly expressed. Furthermore, staining for SRD5A1 revealed punctate signal in neocortical regions of dorsal organoids, primarily in radial glia, but almost no SRD5A1 puncta in ventral organoids (Fig. 5f). This difference in SRD5A1 presence indicates that these two brain regions (dorsal and ventral) might have a different natural aptitude to respond to androgens during in vivo brain development.

\section{Additional progenitors lead to an increased number of neurons}

The enrichment for ontology terms associated with brain size (Fig.

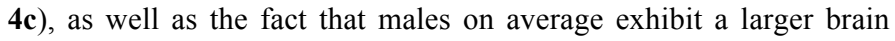
size, pointed to a potential role for effects of androgens on brain size and neuron number. However, we did not observe any change in neuronal numbers upon androgen treatment despite the increase in basal progenitors (Fig. 6a-c). We hypothesised that, due to the continuous nature of these treatments, the progenitors were
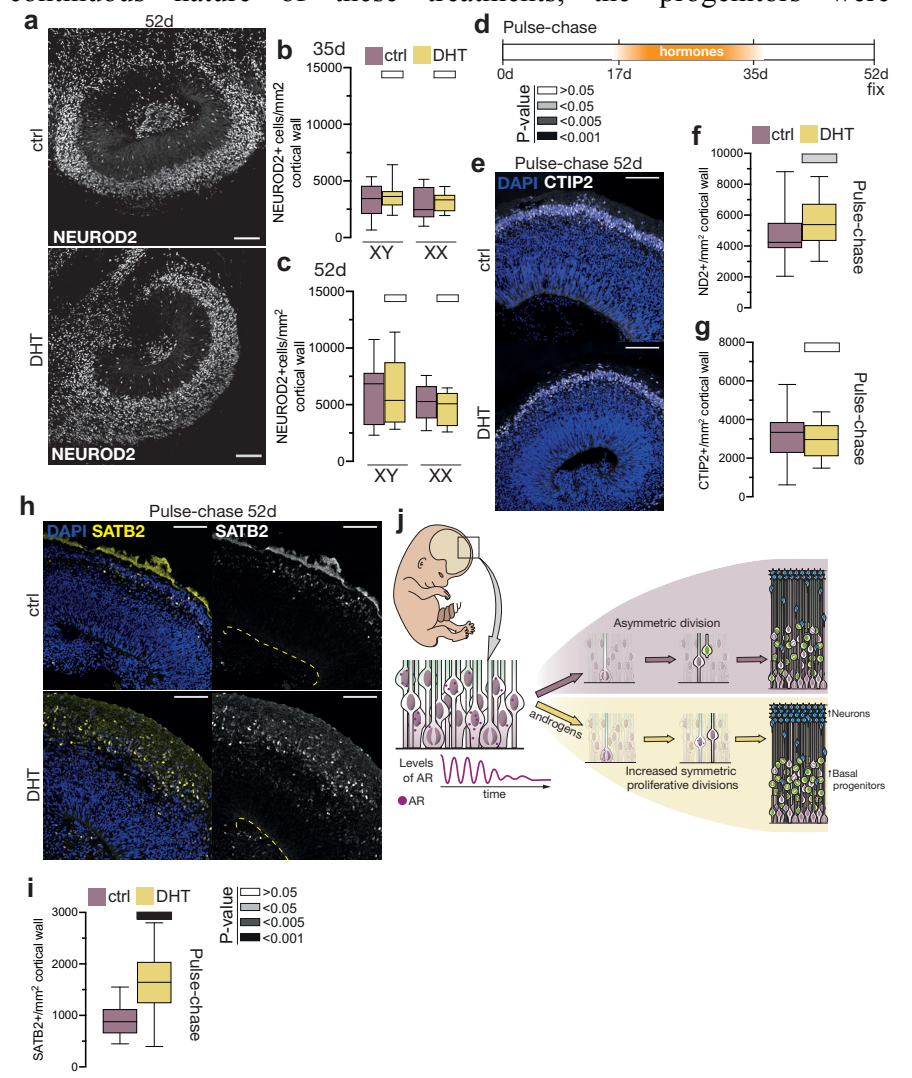

Figure 6. Androgens cause an increase in neuronal numbers

a) Immunostaining of XX 52d, control and DHT-treated, organoids stained for NEUROD2 (white). b) Quantification of NEUROD2+ cells per $\mathrm{mm}^{2}$ of cortical wall at 35 days, in XY and XX organoids. c) Quantification of NEUROD2+ cells per $\mathrm{mm}^{2}$ of cortical wall at 52 days, in XY and XX organoids. d) Timeline of the pulse-chase experiment. Hormones were administered between 17-35d, then washed out and cultured in media alone. Organoids were fixed at 52d. e) Quantification of NEUROD2+ cells per $\mathrm{mm}^{2}$ cortical wall after pulse-chase treatment. f) Immunostaining for CTIP2 (white) of XX 52d organoids after pulse-chase treatment (control and DHT). Co-stained with DAPI (blue). g) Quantification of CTIP2+ cells per $\mathrm{mm}^{2}$ cortical wall after pulse-chase treatment. h) Immunostaining for SATB2 (yellow, white) of XX 52d organoids after pulse-chase treatment. Co-stained with DAPI (blue). Yellow dashed line indicates the ventricular surface. i) Quantification of SATB2+ cells per $\mathrm{mm}^{2}$ cortical wall after pulse-chase treatment. j) A proposed model of the effects of androgens on the developing neocortex. Androgen receptor is present in radial glia during embryonic brain development, but oscillates during the cell cycle and its levels progressively get lower as development progresses. At the onset of neurogenesis, androgens are secreted by the male embryo's testes and act on the division mode of radial glia. Radial glia under the influence of androgens exhibit increased propensity to undergo symmetric proliferative divisions, rather than asymmetric ones, hence increasing the stem cell pool. With the levels of AR dropping over time, effects of androgens weaken and an increased pool of radial glia gives rise to an increase in basal progenitors. Finally, an overall increase in basal progenitors results in more excitatory neurons. Scale bars: a), h) $100 \mu \mathrm{m}, \mathrm{e})$ $50 \mu \mathrm{m}$. Whiskers in boxplots are min-max values. Significance values (Mann-Whitney, two tailed) of the treatment, as compared to the control, indicated by different shades of grey. 
maintained in a proliferative state. We therefore performed a pulsechase experiment in which organoids were subjected to hormone for 18 days (Fig. 6d), followed by their removal from the media until fixation 17 days later. Analysis of neuronal composition showed a subtle, but significant increase in NEUROD2+ neurons upon DHT treatment (Fig. 6e). However, staining for the subset of neurons that make up the early-born deep-layers using the marker CTIP2 revealed no change (Fig. 6f, g). We hypothesised that this may be due to androgen-mediated maintenance of progenitor proliferation during the stage at which these neurons would normally be produced, whereas later born neurons may be expected to show an increase upon their "release" from this proliferative state, after the removal of androgen. Indeed, staining for the upper-layer marker, SATB2, revealed a striking increase in these later born neurons (Fig. 6h, i). These findings demonstrate that an androgen-induced switch towards more proliferative divisions of neural progenitors results in a later increase in excitatory neuron numbers within the neocortex.

\section{Discussion}

Because sex-based brain differences have remained controversial, we sought to provide some clarity to this issue by examining this question from a cell biological and mechanistic standpoint. We examined whether sex-based differences could be observed during human brain development using cerebral organoids as a model system. Although even the most well-established sex-based brain differences are subtle, we were able to observe significant differences in neural progenitor numbers and division modes under the influence of androgens, that overall suggest a shift towards proliferative divisions of radial glial stem cells. These increased stem cells are then able to produce a higher number of basal progenitors, and eventually increased neuron numbers (Fig. 6j).

The fact that we saw highly similar effects of androgens in both male and female brain organoids points to primarily cell-extrinsic driven sex differences in cellular response at this stage of development. This would also fit with the non-binary nature of brain sex-related phenotypes, since a more quantitative (rather than qualitative) difference would emerge from changes in concentrations and timing of sex hormones. Such fine-tuning may also come from the responsiveness of the cells, and our findings of transitory expression of AR in radial glia would support that notion. Thus, while androgens seem to have a general proliferative effect on neural progenitors of the dorsal neocortex, the subtle, cell stage-dependent, and dynamic nature of the androgen response calls for future studies.

In rodents, androgens themselves are not thought to impact neurogenesis during development, although during adult neurogenesis androgens were shown to promote survival of new neurons ${ }^{64}$. Instead, it is estradiol that influences rodent neural progenitors ${ }^{14}$ and masculinises the brain ${ }^{13,65,66}$. However, we observed that in human brain organoids, administration of estradiol caused only minor changes. This discrepancy indicates that either estradiol causes phenotypes that we were not able to observe using this set of parameters, or the timing of these studies is not appropriate for investigation of the role(s) of estrogens. It could also point to human, or perhaps more widely primate, specific biological processes that require further investigation.

The identified androgen regulated genes point to chromatin remodelling and HDAC activity, and our functional studies further support the role of HDACs in mediating the effects of androgens on neural progenitors of the developing human neocortex. HDAC activity has been linked to ASD and schizophrenia, both of which also exhibit a sex-bias and present with sex-differential manifestations.
While prenatal sex hormone levels have been primarily examined in connection with $\mathrm{ASD}^{15,67}$, some reports have shown an association of prenatal androgens and the development of schizophrenia ${ }^{68}$. Our findings connect these risk factors, and suggest that the activity of androgens on HDACs in the developing human neocortex, through their previously established pro-proliferative effects ${ }^{33,58,69,70}$, may help explain the sex-bias of these disorders.

Sex biased neurological and mental health disorder susceptibility suggests the presence of morphological and/or functional differences between healthy male and female brains that may predispose to certain conditions. The most conclusive differences are quantitative in nature, namely brain size $e^{7,9,10,71}$, and our findings of increased cortical excitatory neurons upon androgen treatment would suggest that such differences may, at least in part, arise due to developmental regulation of progenitor behaviour. Interestingly, we did not observe such an effect of androgens in the area that produces inhibitory neurons, pointing to an important role in regulating the balance of excitatory to inhibitory neurons. Along these lines, ASD and schizophrenia seem to share a pathophysiological mechanism of excitatory to inhibitory neuron imbalance ${ }^{59,72-74}$. Thus, our findings are able to consolidate these various observations and suggest that male sex hormones act through HDAC activity to regulate excitatory neuron number and hence brain size. Misregulation of this process could affect the balance between the numbers of excitatory and inhibitory neurons and thus may impact risk for developing sex-biased disorders such as ASD and schizophrenia.

\section{Materials and Methods}

\section{Stem cell maintenance and organoid generation}

Male (XY) and female (XX) stem cells (male - H1 (WiCell WA01) and female - H9 (WiCell WA09)) were approved for use in the project by the UK Stem Cell Bank Steering Committee. Cells were cultured on Matrigel ${ }^{\circledR}$ (Corning, 356234) - coated plates and maintained in StemFlex ${ }^{\text {TM }}$ medium (Thermo Fisher, A3349401). We used the commercial kit (STEMdiff ${ }^{\mathrm{TM}}$ Cerebral Organoid Kit, StemCell Technologies 08570) with previously described modifications to increase embryoid body surface area ${ }^{75}$, namely microscaffolds $(18000$ cells seeded) or smaller EBs (2000 cells seeded). Organoids were transferred to the orbital shaker at $57 \mathrm{rpm}(\phi 25 \mathrm{~cm})$ at $15 \mathrm{~d}( \pm 1$ day, depending on the morphology). Matrigel ${ }^{\circledR}$ was removed manually between days 14-17, after visual inspection. Organoids were transferred to IDM+A supplemented with diluted Matrigel ${ }^{\circledR}$ on day 30 and kept at those conditions until fixation. Ventral organoids were generated as previously described ${ }^{76}$, with ventralizing factors $(2.5 \mu \mathrm{M}$ IWP-2, Sigma, I0536; 100nM SAG, Sigma, 566661) supplemented in the neural induction (NI) medium for 2 days.

\section{Hormone and drug treatments}

All steroid hormones were diluted in $96 \%$ ethanol $(\mathrm{EtOH})$ to obtain stock solution. Organoids were treated with $30 \mathrm{nM}$ or $100 \mathrm{nM}$ dihydrotestosterone (DHT, Sigma A8380-1G), 100nM testosterone (Sigma, T1500), 100nM estradiol (active form of estrogen) (Sigma, E1024) and 100nM progesterone (Sigma, P8783). 96\% ethanol was used as a vehicle control $(1.4 \mu \mathrm{l}$ of $1: 10$ ethanol in PBS to $5 \mathrm{~mL}$ of media, which does not impact the normal development of the neuroepithelium). The treatments started on day 17 of the protocol. Hormone treatments were administered twice a week, with changing of the media. Organoids were collected for analysis at 35 and 52 days. After establishing the phenotype in both cell lines, and since we observed no significant difference between cell lines in their response to different hormones (two-way ANOVA $P=0.23$ ), we focused on the female cell line for subsequent experiments, as it showed greater batch-to-batch consistency. 
Organoids exposed to $100 \mathrm{nM}$ DHT for prolonged periods of time exhibited impaired morphology at later stages (increased cell death and general cytoarchitectural disturbances, data not shown), so we reduced the amount of DHT to $30 \mathrm{nM}$. This amount was chosen because the potency of DHT is roughly three times that of testosterone ${ }^{77}$, meaning any effects caused by $100 \mathrm{nM} \mathrm{T}$ and $30 \mathrm{nM}$ DHT would be comparable.

The application of progesterone to the organoids did not yield any observable phenotype (data not shown). This also eliminated the possibility that progesterone present in the commercially available media (present at $20 \mathrm{nM}$ in N2 and B27 supplements, which are routinely added to the organoid media) might have an effect on organoid development.

For continuous hormone treatments (Sendai/Lenti virus labelling experiments), hormone treatments started immediately after labelling (day 30 or day 45) and were administered every day with the change of media.

Treatment with valproic acid (VPA) (sodium valproate; Stratech, S1168-SEL-200mg) at $0.7 \mathrm{mM}$ was started at $17 \mathrm{~d}$ and continued through until $35 \mathrm{~d}$, with fresh drug supplementation twice a week during media change. DHT was added at $30 \mathrm{nM}$ in the VPA+DHT experiment. Organoids were collected for analysis at $35 \mathrm{~d}$.

The pulse-chase experiment was started at 17 days, and hormones were removed from the media at 35 days. Organoids were then grown in IDM+A with dissolved Matrigel ${ }^{\circledR}$ and fixed at 52 days.

\section{Sendai and Lenti virus labelling}

Organoids were labelled using a CytoTune ${ }^{\mathrm{TM}}$ EmGFP Sendai Fluorescence Reporter (ThermoFisher, A16519). The stock solution of the virus was diluted with PBS to 1:10 and kept on ice. The solution was visualized with Fast Green FCF (Sigma, F7252) and was delivered to the ventricles at $45 \mathrm{~d}$ using a pulled capillary. Organoids were immediately subjected to hormone treatment. The media was changed every day. Organoids were collected for analysis at 6- and 8days post-labelling. At 8 days post labelling, some of the organoids were injected with LentiBrite ${ }^{\mathrm{TM}}$ RFP Control Lentiviral Biosensor (Millipore, 17-10409), using the same procedure as with EmGFP Sendai virus. These organoids were kept in IDM+A with dissolved Matrigel ${ }^{\circledR}$ for a further 8 days and then fixed for clonal analysis.

\section{Plasmids}

Constitutively active androgen receptor (EGFP-C1-AR-V7) ${ }^{26}$ was obtained from Michael Mancini and Marco Marcelli (Addgene \#86856). A plasmid containing estrogen receptor alpha (pEGFP-C1$\mathrm{ER} \alpha$ ) was obtained from Michael Mancini (Addgene \#28230) and mutated into a constitutively active form (pEGFP-C1-ER $\alpha$ Y 537S) ${ }^{29}$ using the Q5 ${ }^{\circledR}$ Site-Directed Mutagenesis Kit (New England Biolabs, E054S).

\section{Electroporations}

Organoids were injected and electroporated at $45 \mathrm{~d}$ as described previously ${ }^{16}$ with the following parameters: voltage $-40 \mathrm{mV}$, duration $-50 \mathrm{~ms}$, pulses -5 , interval $-1.0 \mathrm{~s}$. All plasmids were used at $1 \mu \mathrm{g} / \mathrm{mL}$. EGFP-C1 plasmid was used as a control. Organoids were collected 2and 5-days post-electroporation and media was changed at 2 days post-electroporation.

\section{Organoid fixation and processing}

Organoids were collected at different stages and fixed in 4\% PFA in phosphate buffer (PB) for 1 hour at room temperature (RT). After washing in PBS $(2 \times)$, organoids were infiltrated with $30 \%$ sucrose in
$\mathrm{PB}$ at $4{ }^{\circ} \mathrm{C}$ until the tissue sank. After embedding in $7.5 \%$ gelatin $/ 30 \%$ sucrose in $\mathrm{PB}$ and freezing in isopentane at $-40^{\circ} \mathrm{C}$, tissue blocks were stored at $-80^{\circ} \mathrm{C}$ until sectioning. Organoids were sectioned using a cryostat (Leica, CM1950) at $20 \mu \mathrm{m}$ and sections were collected on coated slides (ThermoFisher Superfrost ${ }^{\circledR}$ Plus, J1800AMNZ). Sections were stored at $-20^{\circ} \mathrm{C}$ until further processing.

\section{Immunohistochemistry and RNA Scope}

Immunostaining was performed as follows: Slides were briefly washed in PBS. Antigen retrieval (if necessary) was performed by using $0.01 \mathrm{M}$ sodium citrate, pH6.0. Slides were submerged in the antigen retrieval solution and heated for one hour at $70^{\circ} \mathrm{C}$ in a water bath. After antigen retrieval, slides were left to cool for 20 minutes at RT. After washing with PBS, sections were blocked with $0.25 \%$ Triton-X 100 with $4 \%$ donkey serum in PBS (permeabilization buffer) for $1-2$ hours at RT. Primary antibodies were diluted in $0.1 \%$ Triton-X 100 with 4\% donkey serum in PBS (blocking buffer) and left overnight at $4{ }^{\circ} \mathrm{C}$. The following primary antibodies were used: rat anti-histone H3 (phospho S28) (Abcam, ab10543, 1:500), rat antiCTIP2 (Abcam, ab18465, 1:200), mouse anti-HuC/D (LifeTech, A21271, 1:200), mouse anti-Ki67 (Dako, MIB-1, 1:100), mouse antiDLX2 (Santa Cruz, sc-393879, 1:200), mouse anti-SATB2 (Abcam, ab51502, 1:50), rabbit anti-NEUROD2 (Abcam, ab104430, 1:200), rabbit anti-Ki67 (Abcam, ab15580, 1:200), rabbit anti-NGN2 (Cell Signalling Technologies, D2R3D, 1:200), rabbit anti-HOPX (FL-73) (Santa Cruz, sc-30216, 1:100), chicken anti-GFP (Thermo Fisher, A10262, 1:500), goat anti-SRD5A1 (Abcam, ab110123, 1:200), goat anti-BRN2 (Santa Cruz, sc-6029, 1:200), sheep anti-TBR2 (R\&D Systems, AF6166, 1:200).

The next day, sections were washed with PBS and secondary antibodies coupled to Alexa Fluor fluorescent dyes (ThermoFisher Scientific) were added (diluted in blocking buffer 1:1000) in combination with DAPI (1:1000). Sections were incubated for 1 hour at RT. After PBS washes, slides were mounted in ProLongTM Diamond (ThermoFisher, P36961), left to dry at RT in the dark, and stored at $4^{\circ} \mathrm{C}$ after imaging.

\section{Analysis of AR mRNA}

AR mRNA was detected using the RNAscope ${ }^{\circledR}$ Fluorescent Multiplex Reagent Kit (ACD, 320850). The probe used was Hs-ARO2 (ACD, 522941). The slides were dehydrated using a sequence of increasing concentrations of EtOH (50-100\%). Dehydrated sections were air-dried for 5' at RT. Heat mediated target retrieval was performed in Target retrieval solution for 2 ' at $95^{\circ} \mathrm{C}$. Diluted protease III $(1: 15)$ was applied and incubated for $15^{\prime}$ at $40^{\circ} \mathrm{C}$ in a humidified chamber. After washing 3 times in PBS, pre-heated probe was applied to the slides and hybridised for 2 hours at $40^{\circ} \mathrm{C}$ in a humidified chamber. After hybridisation, the slides were washed in wash buffer twice. AMP 1-FL was applied and incubated for $30^{\prime}$ at $40^{\circ} \mathrm{C}$. Sections were washed twice for 2' in 1x Wash buffer at RT. AMP 2-FL, AMP 3-FL and AMP 4-FL were individually added to the slides and incubated for $15^{\prime}, 30^{\prime}$ and $30^{\prime}$ at $40^{\circ} \mathrm{C}$, respectively, with wash steps in 1x Wash buffer in between (twice for 2' at RT). The slides were washed 3 times in MilliQ water. Permeabilisation buffer $(0.4 \%$ Triton-X, 4\% normal donkey serum in PBS) was applied and incubated for 1 hour at RT. Primary antibody (diluted in blocking buffer) was incubated over night at RT in a humidified chamber. The following day, slides were washed 3 times for 5' in PBS. Secondary antibody and DAPI were incubated for 2 hours at RT. Slides were washed in PBS 3 times for 5' and mounted in ProLong ${ }^{\text {TM }}$ Diamond.

For analysis of AR mRNA after cell division, $\mathrm{XX}$ organoids were injected with EmGFP Sendai Fluorescence Reporter at 50d as described above to trace individual radial glia stem cells and their 
progeny. Organoids were fixed at $54 \mathrm{~d}$ and submitted to the RNA Scope protocol. The AR mRNA signal was analysed only in welldefined GFP+ pairs of cells. Only AR mRNA puncta that were inside the GFP signal were taken into consideration.

\section{Imaging and image analysis}

Imaging was performed either by using a Panoramic Confocal slide scanner, or Zeiss $710,780 \mathrm{UV}$ or 880 confocal microscopes. Objectives used: $10 \times, 20 \times, 40 \times$ and $64 \times$. Images were taken as either Z-stacks, or individual planes. Quantification of data from all systems were compared to ensure reproducibility of the data. Images obtained were processed using $\mathrm{Fiji}^{78}$.

\section{Quantifications}

All quantifications were done using Fiji. Manual cell counting was performed by a custom plug-in, written by Johannes Schindelin. Only ventricles of appropriate morphology (distinct progenitor and neuronal layers, see below) and healthy appearance were chosen for quantifications.

\section{Cell type, morphology and proliferation quantifications}

The ventricular length was defined as an uninterrupted stretch of apical surface populated by apical progenitors, as shown by phosphohistone H3 (PH3) or an apical progenitor marker staining (PAX6 or SOX2, not shown). The ventricular zone (VZ) was defined as a layer of cells directly abutting the ventricle, with a distinctive radial organisation of nuclei and high density of cells, as revealed by DAPI staining. The subventricular zone (SVZ) was defined as a zone basally adjacent to the VZ, with cells expressing TBR2 and nuclei of a rounder shape. Progenitor zone was defined as the combined area of the ventricular (VZ) and subventricular zone (SVZ) expressing the progenitor marker TBR2. The cortical wall was defined as the area between the apical and the basal side of the cortical lobule. The cortical plate was recognised as a zone at the basal side of the cortical wall containing neurons arranged in an ordered fashion. All counts were normalized per $\mathrm{mm}^{2}$, unless indicated otherwise. Counts were done without using pseudocolour. Quantifications are represented as boxplots. The body of the boxplot represents the $25^{\text {th }}$ to the $75^{\text {th }}$ percentile of the data. The line represents the median. Whiskers represent the minimum and maximum. All quantifications were done on a minimum of three independent organoid batches, with a minimum of 5 ventricles per treatment, per batch. Only live cells were counted. When quantifying multiple stainings, the cell was scored as double positive only if the signal from both channels analysed clearly corresponded in shape.

\section{Clonal analysis}

Only cells labelled GFP- (labelled with CytoTune ${ }^{\mathrm{TM}}$ EmGFP Sendai Fluorescence Reporter) or RFP-positive (labelled with LentiBrite ${ }^{\mathrm{TM}}$ RFP Control Lentiviral Biosensor) were analysed. During imaging, special care was taken to collect all the GFP- or RFP-expressing cells from the $20 \mu \mathrm{m}$ section in the form of a Z-stack. Only clones consisting of $>2$ cells were taken into account, to ensure that the cell had divided upon receiving the virus. Only isolated clones were counted, in order to minimize the probability of several founder cells being infected upon the delivery of the virus, and their clonal progeny intermingling. Measurements were done on a minimum of 3 independent organoid batches, with a minimum of 2 organoids per timepoint, with a minimum of 5 clones analysed per timepoint, per treatment, per batch.

\section{Quantification of SRD5A1 puncta}

After immunostaining with anti-SRD5A1 antibody, images were obtained using a $63 \times$ objective, with exactly the same laser power, exposure time and digital gain for both the control and DHT-treated sections. A square measuring $100 \times 100 \mu \mathrm{m}$ was designated in the VZ. Threshold was adjusted in order to remove any immunostaining background which was easily discernible from the SRD5A1 signal. SRD5A1 puncta were then automatically counted using the "Analyze particles" option.

\section{Live imaging}

Organoids were electroporated at day 31 or 54 , as described above. Plasmids containing pCAG-mCherry and EGFP-C1-AR-V7 were mixed to $300 \mathrm{ng} / \mathrm{mL}$ and $1 \mu \mathrm{g} / \mathrm{mL}$, respectively, and delivered to the ventricles. Electroporated organoids were transferred to IDM+A+Matrigel ${ }^{\circledR}$ medium for several hours for recovery. After that, organoids were embedded in 3\% Low melting agarose (Sigma, A9414) in HBSS (1×) (Gibco, 14025092), left to settle and sectioned on the vibratome at $300 \mu \mathrm{m}$. Sections were placed on Millicell Organotypic Cell Culture Inserts (Merck, PICM0RG50) and supplemented with $800 \mu 1$ serum-free media ${ }^{79}$. After 24 hours at $37^{\circ} \mathrm{C}$, slices were imaged using Zeiss 880 Airy Scan, with the following parametres: $20 \times$ objective, FAST scanning mode, 1072x1072 pixels, scanning interval of $10-11.45$ minutes, total imaging time $>25$ hours. Movies were processed using Zeiss ZEN software and Fiji.

\section{Western blot}

Organoids for Western blot were treated with $30 \mathrm{nM}$ DHT or $100 \mathrm{nM} \mathrm{E}$ every day, starting from d17 and collected every two days. Organoids ( 7 to 10 per condition) were washed twice in PBS prior to snapfreezing in liquid nitrogen. $\mathrm{XX}$ stem cells were used as a positive control, and XX d75 organoids as a negative (due to very low AR expression at this stage). Organoids and cells were lysed in modified RIPA buffer (mRIPA: 1\% Triton-X, 0.1\% SDS, $150 \mathrm{mM} \mathrm{NaCl}, 50 \mathrm{mM}$ Tris $\mathrm{pH} 7.4,2 \mathrm{mM}$ EDTA, $12 \mathrm{mM}$ sodium deoxycholate) supplemented with protease (Thermo Fisher, 78430) and phosphatase (SigmaAldrich, 4906845001) inhibitors. Quick Start Bradford Dye Reagent (Bio-Rad, 5000205) assay was used to determine protein concentration. $20 \mu \mathrm{g}$ of total protein was loaded for every sample except for the negative control where $10 \mu \mathrm{g}$ of protein were loaded. Samples were run on SDS polyacrylamide gel electrophoresis (SDSPAGE) $4-20 \%$ Tris glycine gel at $90 \mathrm{~V}$ in MOPS-SDS buffer and transferred overnight at $30 \mathrm{~V}$ to Amersham Hybond P 0.45 PVDF blotting membranes (GE Healthcare, 10600023). Membranes were blocked in 5\% BSA for 2 hours at RT and incubated overnight with the following primary antibodies, rabbit anti-AR (Cell Signalling, D6F11, 1:2000), mouse anti-GAPDH (Abcam, ab8245, 1:2000). Membranes were washed in TBST buffer prior to incubation with secondary fluorophore conjugated antibodies $1: 2000$ for $1 \mathrm{~h}$ at RT (goat anti-mouse DyLight 680 Invitrogen A21058, goat anti-rabbit DyLight 800 CST 5151). Membranes were washed in TBST and imaged using a Li-COR Odyssey CLx Infrared Imaging System.

\section{RNA-Seq library preparation and sequencing}

Organoids were generated from the XX cell line and treated as described above (hormone treatment started at 17d). Organoids were collected at $35 \mathrm{~d}$, snap-frozen in liquid nitrogen and stored at $-80^{\circ} \mathrm{C}$ until processing. Two to three individual organoids were collected per treatment. Total RNA from individual organoids was isolated using the RNeasy Mini Kit (Qiagen, 74104). Genomic DNA was removed using the Invitrogen $^{\mathrm{TM}}$ TURBO DNA-free ${ }^{\mathrm{TM}}$ kit (Invitrogen, AM1907). After isolation, total RNA from individual organoids of the same treatment was pooled for each library preparation. RNA quality was assessed using Bioanalyzer (Agilent, G2939BA). Libraries were prepared using the NEBNext ${ }^{\circledR}$ Ultra II DNA Library Prep Kit for Illumina ${ }^{\circledR}$ (New England BioLabs, E7645) with starting input of 500 ng of total RNA per sample. Ribosomal RNA was removed and mRNA isolated using the $\operatorname{NEBNext}{ }^{\circledR}$ Poly(A) mRNA Magnetic 
Isolation Module (New England BioLabs, E7490). During library preparation, AMPure XP Beads (Beckman Coulter, A63881) were used for reaction clean ups. NEBNext ${ }^{\circledR}$ Multiplex Oligos (dual index primers) (New England BioLabs, E7600) were used in the PCR enrichment step, with 11 cycles. Library quality was assessed by Bioanalyzer and the concentration was determined using the NEBNext ${ }^{\circledR}$ Library Quant Kit for Illumina ${ }^{\circledR}$ (New England BioLabs, E7630). Libraries were pooled and sequenced on the HiSeq4000 machine (Illumina) at 50 base pair length, single ended, to a minimum of 30 million reads per library.

\section{Data analysis and statistics}

Analysis of cell number quantification was performed using GraphPad Prism 8 software. Statistical analyses of control versus hormone treatments were done as multiple pairwise analyses, using unpaired non-parametric test (Mann-Whitney, two-tailed) with significance threshold of $\mathrm{P}<0.05$.

FASTQ sequence read files were aligned to the hg38 transcriptome (with decoy sequences) and quantified using salmon v0.14.1 ${ }^{80}$ applying both a sequence bias correction and a GC bias correction with a mean fragment length of $300 \mathrm{bp}$ and a standard deviation of 50 bp. Salmon quantification files containing Transcripts Per Million (TPM) estimates were imported into R using tximport v1.16.1 and DESeq2 v1.28.1 ${ }^{81}$. Prior to analysis, a non-parametric empirical Bayesian batch-correction was applied to the data using the function 'ComBat' in sva v3.36.0 (Extended Data Fig. 5a, b), and only genes with $>4$ TPM on average per sample ( 6 androgen (DHT/T), 3 control) were retained for downstream analysis.

Differential expression analysis was conducted using a novel approach $^{31}$ that employs a logistic elastic-net regression implemented in the R package glmnet $\mathrm{v} 4.0-2^{83}$ using an alpha of 0.5 . The response variable was taken to be the sample hormone status (Androgen or Control), and the independent variable was the transcript abundance estimates for individual genes. To determine the value of lambda - the parameter controlling the strength of the regression penalty - for selecting differentially expressed genes, cross-validation was performed using the function 'cv.glmnet' and the 'type.measure' argument set to 'deviance'. The value of lambda that minimised the deviance (a measure of goodness-of-fit) of the model was selected, and genes with a non-zero regression coefficient at the selected lambda value were considered to be differentially expressed, which included both up- and down-regulated genes. Log-fold change estimates for the selected genes were estimated using DESeq2.

Validation of the logistic elastic-net model was carried out using the same input dataset in which a ComBat batch-correction was applied to the treatment variable (Androgen or Control) to remove signal from the dataset. Then, a known amount of log-fold change signal was added to a random sample of genes using the binomial-thinning approach implemented in the R package seqgendiff v1.2.2 $2^{84}$ using the function 'thin_diff' and randomly selecting 6 of the samples to be androgen samples and 3 to be controls. To determine the number of genes to add signal to, the R package locfdr v1.1- ${ }^{85}$ was used to fit a mixture model to DESeq2 raw p-values derived from the original unmodified dataset. This package enables an estimate of the total number of non-null genes in the dataset - in this case 362. 'locfdr' was also used to infer the distribution of log-fold changes for non-null genes using DESeq2 log-fold change estimates derived from the original unmodified dataset. $362 \mathrm{log}$-fold changes were then randomly sampled from this distribution and paired with 362 randomly sampled genes. Both DESeq2 and the elastic-net models were then used to infer differential expression in this modified dataset and both the sensitivity and precision of each method was calculated. While
DESeq 2 achieved $100 \%$ precision, it had a sensitivity of just $2 \%$ (genes with an adjusted p-value $<0.1$ were considered differentially expressed). In contrast, the elastic-net method achieved a sensitivity of $20 \%$ ( 72 correctly called genes) with a precision of $96 \%$ - all three false positives (out of 75 hits) had absolute log fold changes below 0.31 whereas $75 \%$ of the 72 true positive hits had an absolute log fold change above 0.37 . In addition, false negatives had a significantly lower log fold change than true positives (Wilcoxon rank sum test, $\mathrm{p}$ $=3 \mathrm{e}-13)$. A reproducible $\mathrm{R}$ markdown script and data can be accessed at https://github.com/alextkalinka/hormone-DE-analysis.

\section{Acknowledgements}

We would like to thank Deepak Srivastava, Sean Munro and Manu Hegde for helpful comments. We would like to thank the MRC LMB Light Microscopy facility and Bioinformatics (Paula Freire-Pritchett), as well as the CRUK Genomics facility. We would like to thank Michael Mancini, Marco Marcelli and Elizabeth Wilson for depositing their plasmids in Addgene. We thank the other members of the Lancaster lab and the MRC LMB Cell biology division for fruitful discussions. Work in the Lancaster lab is supported by the Medical Research Council (MC_UP_1201/9) and the European Research Council (ERC STG 757710).

\section{Author contributions}

IK conceived the study, performed experiments, analysed data and wrote the paper. IC performed experiments and analysed the data. LP performed experiments. ATK analysed the RNA-seq data. MAL supervised the study and co-wrote the paper.

\section{Declaration of interests}

The authors declare no competing interests.

\section{References}

1. Green, T., Flash, S. \& Reiss, A. L. Sex differences in psychiatric disorders: what we can learn from sex chromosome aneuploidies. Neuropsychopharmacol 44, 9-21 (2018).

2. McCarthy, M. M. Multifaceted origins of sex differences in the brain. Philosophical Transactions Royal Soc B Biological Sci 371, 20150106 (2016).

3. Loomes, R., Hull, L. \& Mandy, W. P. L. What Is the Male-toFemale Ratio in Autism Spectrum Disorder? A Systematic Review and Meta-Analysis. J Am Acad Child Adolesc Psychiatry 56, 466474 (2017).

4. LeGates, T. A., Kvarta, M. D. \& Thompson, S. M. Sex differences in antidepressant efficacy. Neuropsychopharmacol 44, 140-154 (2018).

5. Donner, N. C. \& Lowry, C. A. Sex differences in anxiety and emotional behavior. Pflügers Archiv - European J Physiology 465, 601-626 (2013).

6. Abel, K. M., Drake, R. \& Goldstein, J. M. Sex differences in schizophrenia. Int Rev Psychiatr 22, 417-428 (2010).

7. Ritchie, S. J. et al. Sex Differences in the Adult Human Brain: Evidence from 5216 UK Biobank Participants. Cereb Cortex 28, 2959-2975 (2018).

8. Ruigrok, A. N. V. et al. A meta-analysis of sex differences in human brain structure. Neurosci Biobehav Rev 39, 34-50 (2014).

9. Knickmeyer, R. C. et al. Impact of Sex and Gonadal Steroids on Neonatal Brain Structure. Cereb Cortex 24, 2721-2731 (2014).

10. Gilmore, J. H. et al. Regional Gray Matter Growth, Sexual Dimorphism, and Cerebral Asymmetry in the Neonatal Brain. J Neurosci 27, 1255-1260 (2007). 
11. Arnold, A. P. A general theory of sexual differentiation. J Neurosci Res 95, 291-300 (2017).

12. Arnold, A. P. The organizational-activational hypothesis as the foundation for a unified theory of sexual differentiation of all mammalian tissues. Horm Behav 55, 570-578 (2009).

13. McCarthy, M. M. Estradiol and the Developing Brain. Physiol Rev 88, 91-134 (2008).

14. Martínez-Cerdeño, V., Noctor, S. C. \& Kriegstein, A. R. Estradiol stimulates progenitor cell division in the ventricular and subventricular zones of the embryonic neocortex. Eur J Neurosci 24, 3475-3488 (2006).

15. Quartier, A. et al. Genes and Pathways Regulated by Androgens in Human Neural Cells, Potential Candidates for the Male Excess in Autism Spectrum Disorder. Biol Psychiat 84, 239-252 (2018).

16. Lancaster, M. A. et al. Cerebral organoids model human brain development and microcephaly. Nature 501, 373 (2013).

17. Camp, J. G. et al. Human cerebral organoids recapitulate gene expression programs of fetal neocortex development. Proc National Acad Sci 112, 15672-15677 (2015).

18. Miller, W. L. \& Auchus, R. J. The Molecular Biology, Biochemistry, and Physiology of Human Steroidogenesis and Its Disorders. Endocr Rev 32, 81-151 (2011).

19. Hines, M., Constantinescu, M. \& Spencer, D. Early androgen exposure and human gender development. Biol Sex Differ 6, 3 (2015).

20. Pollen, A. A. et al. Molecular Identity of Human Outer Radial Glia during Cortical Development. Cell 163, 55-67 (2015).

21. Vaid, S. et al. A novel population of Hopx-dependent basal radial glial cells in the developing mouse neocortex. Development 145, dev.169276 (2018).

22. Davey, R. A. \& Grossmann, M. Androgen Receptor Structure, Function and Biology: From Bench to Bedside. Clin Biochem Rev Australian Assoc Clin Biochem 37, 3-15 (2016).

23. Pomerantz, S. M. \& Sholl, S. A. Analysis of sex and regional differences in androgen receptors in fetal rhesus monkey brain. Dev Brain Res 36, 151-154 (1987).

24. Wang, F. et al. RNAscope: a novel in situ RNA analysis platform for formalin-fixed, paraffin-embedded tissues. J Mol Diagnostics Jmd 14, 22-9 (2012).

25. Dietrich, W. et al. Testosterone dependent androgen receptor stabilization and activation of cell proliferation in primary human myometrial microvascular endothelial cells. Fertil Steril 95, 12471255.e2 (2011).

26. Qu, Y. et al. Constitutively Active AR-V7 Plays an Essential Role in the Development and Progression of Castration-Resistant Prostate Cancer. Sci Rep-uk 5, 7654 (2015).

27. Bishop, J. L. et al. The Master Neural Transcription Factor BRN2 Is an Androgen Receptor-Suppressed Driver of Neuroendocrine Differentiation in Prostate Cancer. Cancer Discov 7, 54-71 (2016).

28. Dominguez, M. H., Ayoub, A. E. \& Rakic, P. POU-III transcription factors (Brn1, Brn2, and Oct6) influence neurogenesis, molecular identity, and migratory destination of upper-layer cells of the cerebral cortex. Cereb Cortex New York N Y 1991 23, 2632-43 (2012).

29. Jeselsohn, R. et al. Emergence of Constitutively Active Estrogen Receptor- Mutations in Pretreated Advanced Estrogen ReceptorPositive Breast Cancer. Clin Cancer Res 20, 1757-1767 (2014).

30. González, M. et al. Distribution patterns of estrogen receptor $\alpha$ and $\beta$ in the human cortex and hippocampus during development and adulthood. J Comp Neurol 503, 790-802 (2007).

31. Kalinka, A. T. Improving the sensitivity of differential-expression analyses for under-powered RNA-seq experiments. bioRxiv (2020) doi:10.1101/2020.10.15.340737.

32. Tang, T. et al. HDAC1 and HDAC2 Regulate Intermediate Progenitor Positioning to Safeguard Neocortical Development.
Neuron 101, 1117-1133.e5 (2019).

33. Li, L., Jin, J. \& Yang, X.-J. Histone deacetylase 3 governs perinatal cerebral development via neural stem and progenitor cells. Iscience 20, 148-167 (2019).

34. Dey, A. et al. YB-1 is elevated in medulloblastoma and drives proliferation in Sonic hedgehog-dependent cerebellar granule neuron progenitor cells and medulloblastoma cells. Oncogene 35, 4256-4268 (2016).

35. Bizzotto, S. et al. Eml1 loss impairs apical progenitor spindle length and soma shape in the developing cerebral cortex. Sci Rep-uk 7, 17308 (2017).

36. Kielar, M. et al. Mutations in Eml1 lead to ectopic progenitors and neuronal heterotopia in mouse and human. Nat Neurosci 17, 923-933 (2014).

37. Zhang, X. et al. Mutations in QARS, Encoding Glutaminyl-tRNA Synthetase, Cause Progressive Microcephaly, Cerebral-Cerebellar Atrophy, and Intractable Seizures. Am J Hum Genetics 94, 547-558 (2014).

38. Sakai, D., Dixon, J., Dixon, M. J. \& Trainor, P. A. Mammalian neurogenesis requires Treacle-Plk1 for precise control of spindle orientation, mitotic progression, and maintenance of neural progenitor cells. Plos Genet 8, e1002566 (2012).

39. Qin, L. et al. Social deficits in Shank3-deficient mouse models of autism are rescued by histone deacetylase (HDAC) inhibition. Nat Neurosci 21, 564-575 (2018).

40. Mazón-Cabrera, R., Vandormael, P. \& Somers, V. Antigenic Targets of Patient and Maternal Autoantibodies in Autism Spectrum Disorder. Front Immunol 10, 1474 (2019).

41. P, S. S. Modifiers of Severity in Autism Spectrum Disorder. J Genetics Genome Res 6, (2019).

42. Fromer, M. et al. Gene expression elucidates functional impact of polygenic risk for schizophrenia. Nat Neurosci 19, 1442-1453 (2016). 43. Raudvere, U. et al. g:Profiler: a web server for functional enrichment analysis and conversions of gene lists (2019 update). Nucleic Acids Res 47, W191-W198 (2019).

44. Murray, E. K., Hien, A., Vries, G. J. de \& Forger, N. G. Epigenetic Control of Sexual Differentiation of the Bed Nucleus of the Stria Terminalis. Endocrinology 150, 4241-4247 (2009).

45. Pinto, D. et al. Convergence of Genes and Cellular Pathways Dysregulated in Autism Spectrum Disorders. Am J Hum Genetics 94, 677-694 (2014).

46. Williams, S. R. et al. Haploinsufficiency of HDAC4 Causes Brachydactyly Mental Retardation Syndrome, with Brachydactyly Type E, Developmental Delays, and Behavioral Problems. Am J Hum Genetics 87, 219-228 (2010).

47. Bahari-Javan, S. et al. HDAC1 links early life stress to schizophrenia-like phenotypes. Proc National Acad Sci 114, E4686E4694 (2017).

48. Gilbert, T. M. et al. PET neuroimaging reveals histone deacetylase dysregulation in schizophrenia. J Clin Invest 129, 364-372 (2018).

49. Nguyen, H. T. et al. Integrated Bayesian analysis of rare exonic variants to identify risk genes for schizophrenia and neurodevelopmental disorders. Genome Med 9, 114 (2017).

50. Udawela, M. et al. SELENBP1 expression in the prefrontal cortex of subjects with schizophrenia. Transl Psychiat 5, e615 (2015).

51. Fatemi, S. H., Folsom, T. D. \& Thuras, P. D. Deficits in GABA(B) receptor system in schizophrenia and mood disorders: a postmortem study. Schizophr Res 128, 37-43 (2011).

52. Narayan, S., Head, S. R., Gilmartin, T. J., Dean, B. \& Thomas, E. A. Evidence for disruption of sphingolipid metabolism in schizophrenia. J Neurosci Res 87, 278-88 (2009).

53. Göttlicher, M. et al. Valproic acid defines a novel class of HDAC inhibitors inducing differentiation of transformed cells. Embo J 20, 6969-6978 (2001).

54. Wide, K., Winbladh, B. \& Källén, B. Major malformations in 
infants exposed to antiepileptic drugs in utero, with emphasis on carbamazepine and valproic acid: a nation-wide, population-based register study. Acta Paediatr 93, 174-176 (2007).

55. Roullet, F. I., Lai, J. K. Y. \& Foster, J. A. In utero exposure to valproic acid and autism - A current review of clinical and animal studies. Neurotoxicol Teratol 36, 47-56 (2013).

56. Fujimura, K., Mitsuhashi, T., Shibata, S., Shimozato, S. \& Takahashi, T. In Utero Exposure to Valproic Acid Induces Neocortical Dysgenesis via Dysregulation of Neural Progenitor Cell Proliferation/Differentiation. J Neurosci 36, 10908-10919 (2016).

57. Iacopino, F. et al. Valproic acid activity in androgen-sensitive and -insensitive human prostate cancer cells. Int J Oncol 32, 1293-1303 (1992).

58. Hagelkruys, A. et al. A single allele of Hdac2 but not Hdac1 is sufficient for normal mouse brain development in the absence of its paralog. Development 141, 604-616 (2014).

59. Sohal, V. S. \& Rubenstein, J. L. R. Excitation-inhibition balance as a framework for investigating mechanisms in neuropsychiatric disorders. Mol Psychiatr 24, 1248-1257 (2019).

60. Rubenstein, J. L. R. \& Merzenich, M. M. Model of autism: increased ratio of excitation/inhibition in key neural systems. Genes Brain Behav 2, 255-267 (2003).

61. Marín, O. \& Müller, U. Lineage origins of GABAergic versus glutamatergic neurons in the neocortex. Curr Opin Neurobiol 26, 132-141 (2014).

62. Rakic, P. Evolution of the neocortex: a perspective from developmental biology. Nat Rev Neurosci 10, 724-735 (2009).

63. O'Shaughnessy, P. J. et al. Alternative (backdoor) androgen production and masculinization in the human fetus. Plos Biol 17, e3000002 (2019).

64. Swift-Gallant, A. et al. Neural androgen receptors affect the number of surviving new neurones in the adult dentate gyrus of male mice. J Neuroendocrinol 30, e12578 (2018).

65. Nugent, B. M. et al. Brain feminization requires active repression of masculinization via DNA methylation. Nat Neurosci 18, 690-697 (2015).

66. McCarthy, M. M. \& Arnold, A. P. Reframing sexual differentiation of the brain. Nat Neurosci 14, 677 (2011).

67. Baron-Cohen, S. et al. Elevated fetal steroidogenic activity in autism. Mol Psychiatr 20, 369 (2015).

68. Collinson, S. L. et al. Increased ratio of 2 nd to 4 th digit (2D:4D) in schizophrenia. Psychiat Res 176, 8-12 (2010).

69. Li, Y. \& Seto, E. HDACs and HDAC Inhibitors in Cancer Development and Therapy. Csh Perspect Med 6, a026831 (2016).

70. Yamaguchi, T. et al. Histone deacetylases 1 and 2 act in concert to promote the G1-to-S progression. Gene Dev 24, 455-469 (2010).

71. Wang, S. et al. Assessment of neonatal brain volume and growth at different postmenstrual ages by conventional MRI. Medicine 97, e11633 (2018)

72. Bruining, $H$. et al. Measurement of excitation-inhibition ratio in autism spectrum disorder using critical brain dynamics. Sci Rep-uk 10, 9195 (2020).

73. Rubenstein, J. L. Three hypotheses for developmental defects that may underlie some forms of autism spectrum disorder. Curr Opin Neurol 23, 118-123 (2010).

74. Zikopoulos, B. \& Barbas, H. Altered neural connectivity in excitatory and inhibitory cortical circuits in autism. Front Hum Neurosci 7, 609 (2013).

75. Lancaster, M. A. et al. Guided self-organization and cortical plate formation in human brain organoids. Nat Biotechnol 35, 659 (2017).

76. Bagley, J. A., Reumann, D., Bian, S., Lévi-Strauss, J. \& Knoblich, J. A. Fused cerebral organoids model interactions between brain regions. Nat Methods 14, 743-751 (2017).

77. Wright, A. S., Thomas, L. N., Douglas, R. C., Lazier, C. B. \& Rittmaster, R. S. Relative potency of testosterone and dihydrotestosterone in preventing atrophy and apoptosis in the prostate of the castrated rat. J Clin Invest 98, 2558-2563 (1996).

78. Schindelin, J. et al. Fiji: an open-source platform for biologicalimage analysis. Nat Methods 9, 676-82 (2012).

79. Giandomenico, S. L. et al. Cerebral organoids at the air-liquid interface generate diverse nerve tracts with functional output. Nat Neurosci 22, 669-679 (2019).

80. Patro, R., Duggal, G., Love, M. I., Irizarry, R. A. \& Kingsford, C. Salmon provides fast and bias-aware quantification of transcript expression. Nat Methods 14, 417-419 (2017).

81. Love, M. I., Huber, W. \& Anders, S. Moderated estimation of fold change and dispersion for RNA-seq data with DESeq2. Genome Biol 15,550 (2014).

82. Leek, J. T., Johnson, W. E., Parker, H. S., Jaffe, A. E. \& Storey, J. D. The sva package for removing batch effects and other unwanted variation in high-throughput experiments. Bioinform Oxf Engl 28, 882-3 (2012).

83. Friedman, J., Hastie, T. \& Tibshirani, R. Regularization Paths for Generalized Linear Models via Coordinate Descent. J Stat Softw 33, $1-22(2010)$.

84. Gerard, D. Data-based RNA-seq simulations by binomial thinning. Bmc Bioinformatics 21, 206 (2020).

85. Efron, B. Size, power and false discovery rates. Arxiv 35, 13511377 (2007). 
bioRxiv preprint doi: https://doi.org/10.1101/2020.10.24.353359; this version posted October 24, 2020. The copyright holder for this preprint (which was not certified by peer review) is the author/funder. All rights reserved. No reuse allowed without permission.

Extended Data Figures

\section{Extended Data Figure 1.}

a

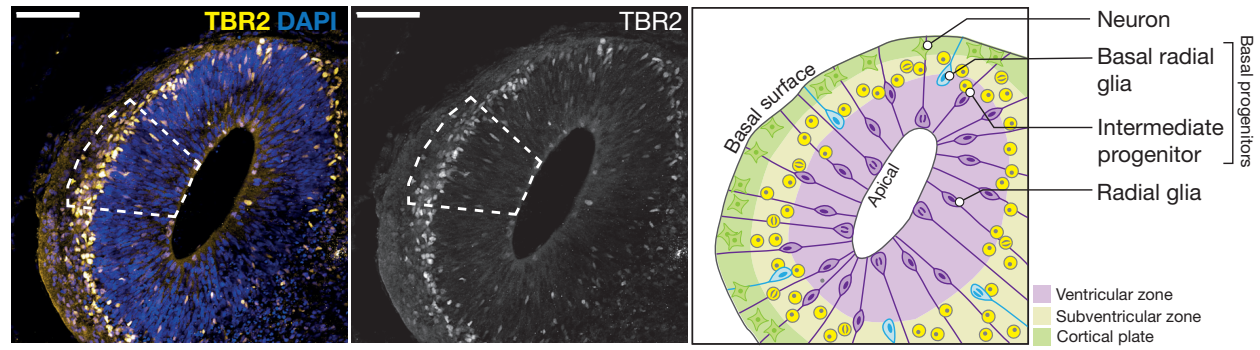

b
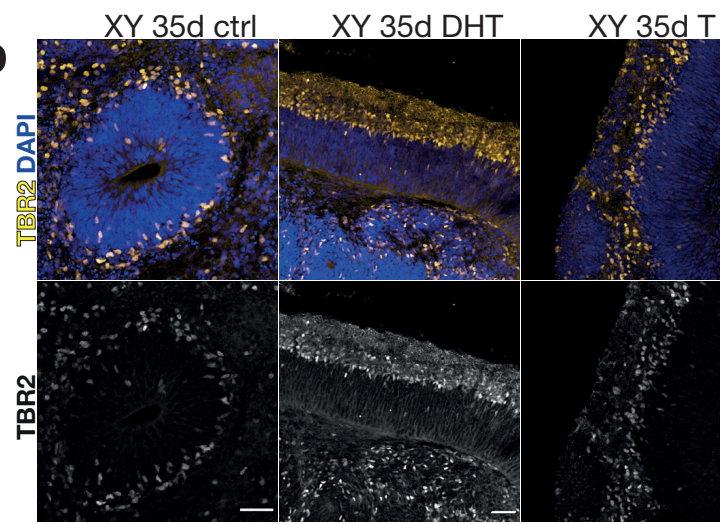

$X Y$ 35d E
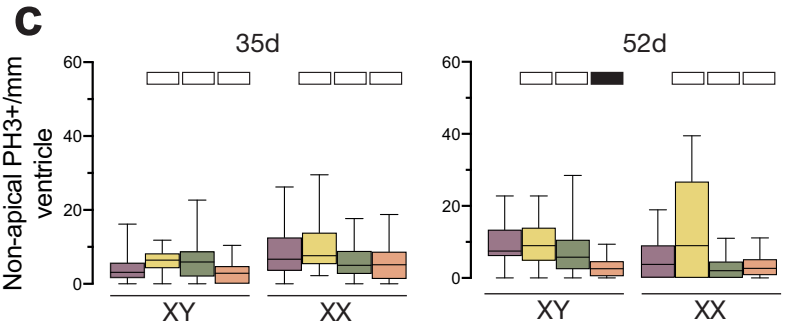

ctrl

DHT

$T$

E

$\stackrel{\Phi}{\frac{1}{3}} \square>0.05$

光 $\square<0.05$

i $0<0.005$

Extended Data Figure 1

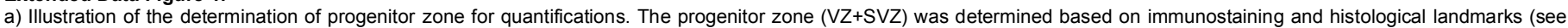

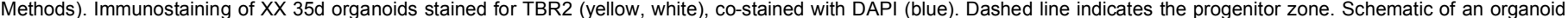

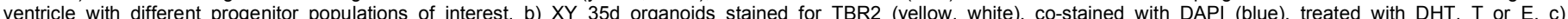

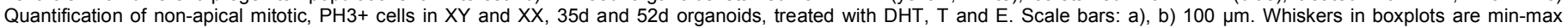
values. Significance values (Mann-Whitney, two tailed) of the treatment, as compared to the control, indicated by different shades of grey. 


\section{Extended Data Figure 2.}

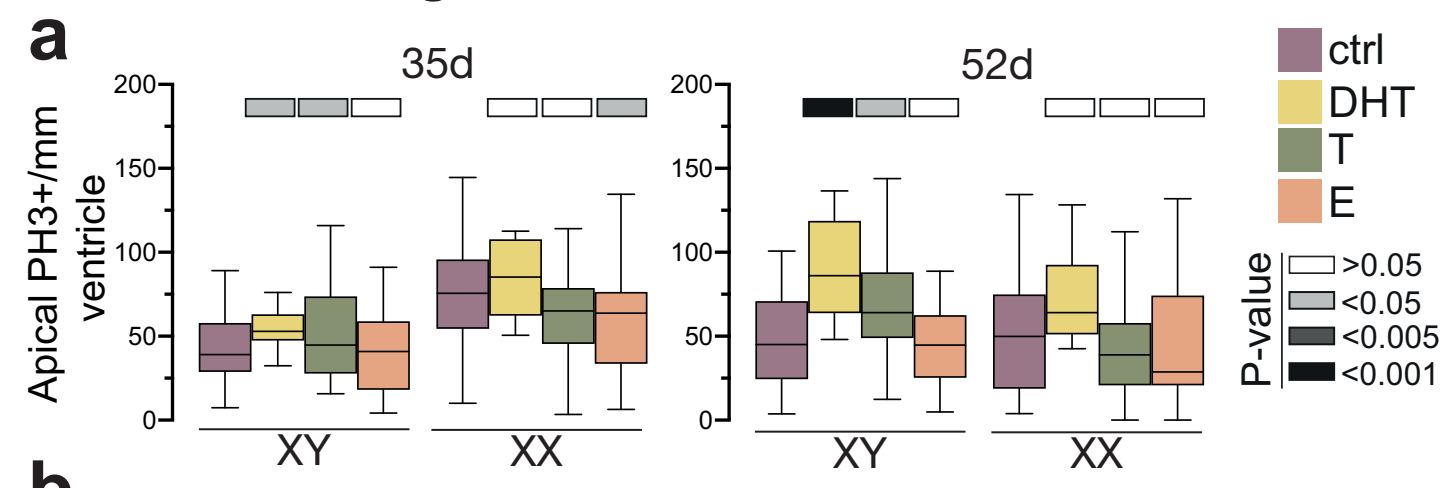

b

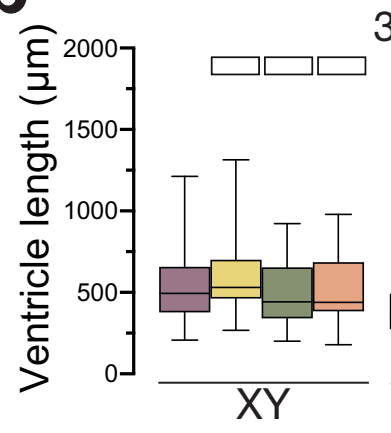

$35 d$

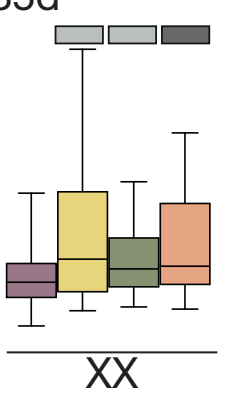

-

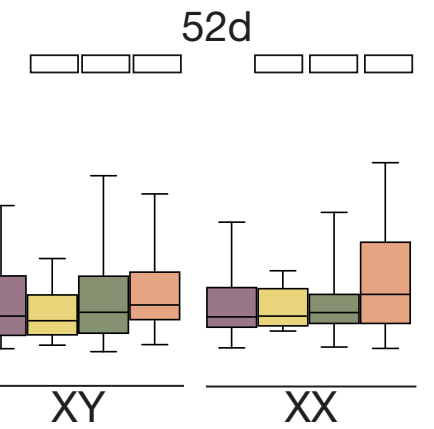

Extended Data Figure 2.

a) Quantification of apical mitotic cells (PH3+), normalized per mm length of ventricle in male (XY) and female (XX) organoids, at 35d and 52d, treated with DHT, T and E. b) Quantification of ventricular length in male (XY) and female (XX) organoids, at 35d and 52d, treated with DHT, T and E. Whiskers in boxplots are min-max values. Significance values (Mann-Whitney, two tailed) of the treatment, as compared to the control, indicated by different shades of grey. 
bioRxiv preprint doi: https://doi.org/10.1101/2020.10.24.353359; this version posted October 24, 2020. The copyright holder for this preprint (which was not certified by peer review) is the author/funder. All rights reserved. No reuse allowed without permission.

\section{Extended Data Figure 3.}

\section{a}

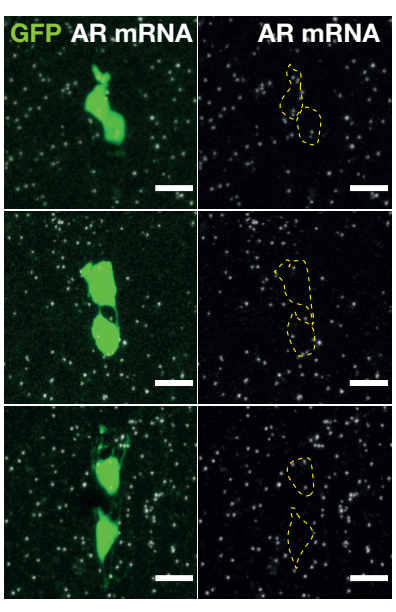

\section{b}

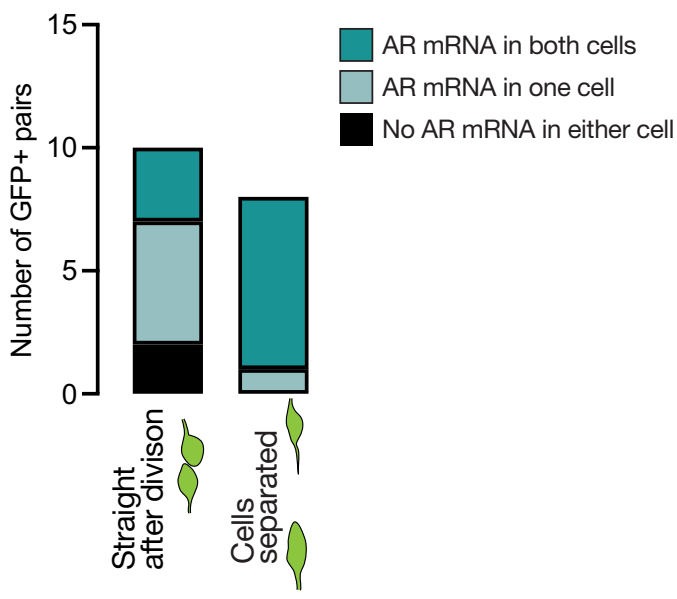

C

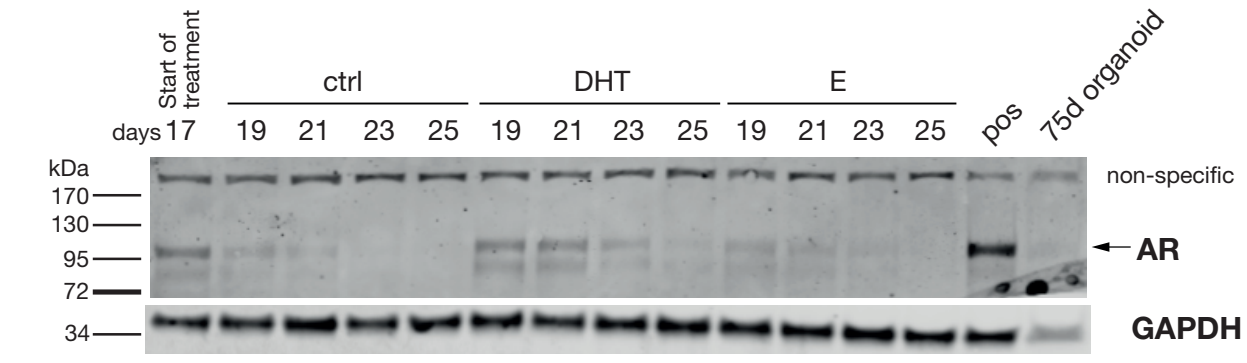

Extended Data Figure 3.

a) RNA Scope of androgen receptor (AR) mRNA (white puncta), together with GFP signal (green) from EmGFP Sendai Fluorescence Reporter showing examples of three daughter cell pairs. Yellow dashed lines indicate GFP+ cell body. b) Quantification of AR mRNA distribution in GFP+ pairs of cells, depending on the stage of cell division. c) Western blot for AR on control, DHT and E treated organoids at 17-25 days. AR specific band is as predicted to be $\sim 110 \mathrm{kDa}$. Note the increased AR signal in DHT-treated organoids. Scale bar: a) $5 \mu \mathrm{m}$. 
bioRxiv preprint doi: https://doi.org/10.1101/2020.10.24.353359; this version posted October 24, 2020. The copyright holder for this preprint (which was not certified by peer review) is the author/funder. All rights reserved. No reuse allowed without permission.

\section{Extended Data Figure 4.}

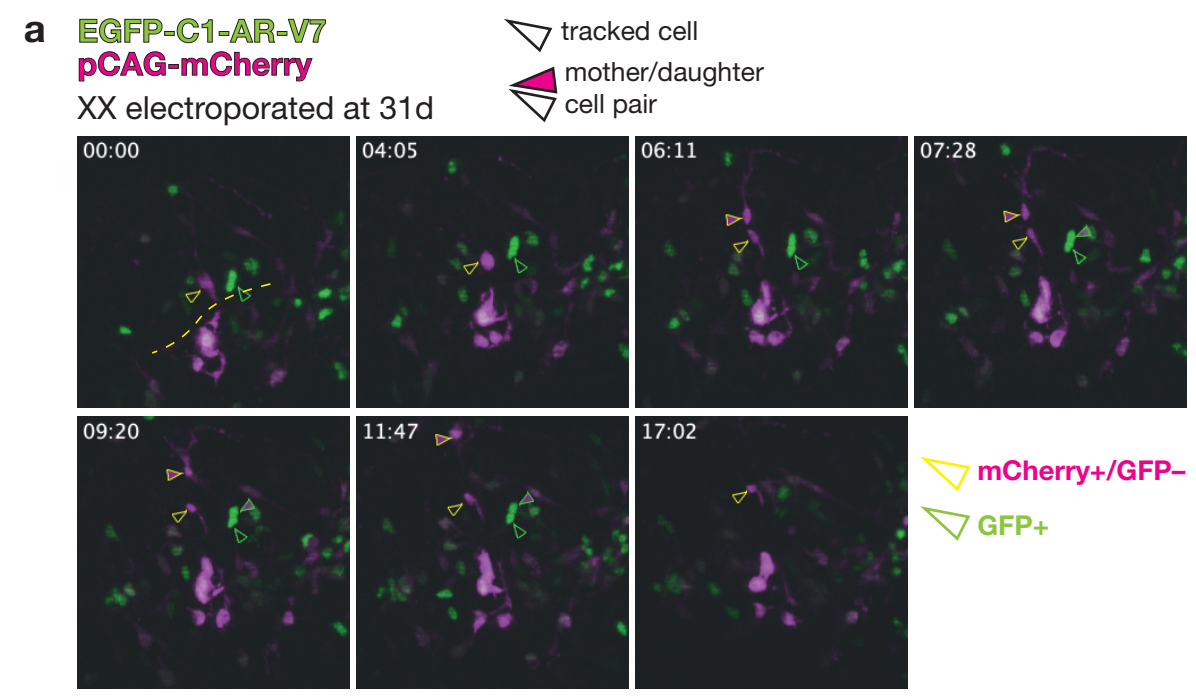

b XX electroporated at $54 d$

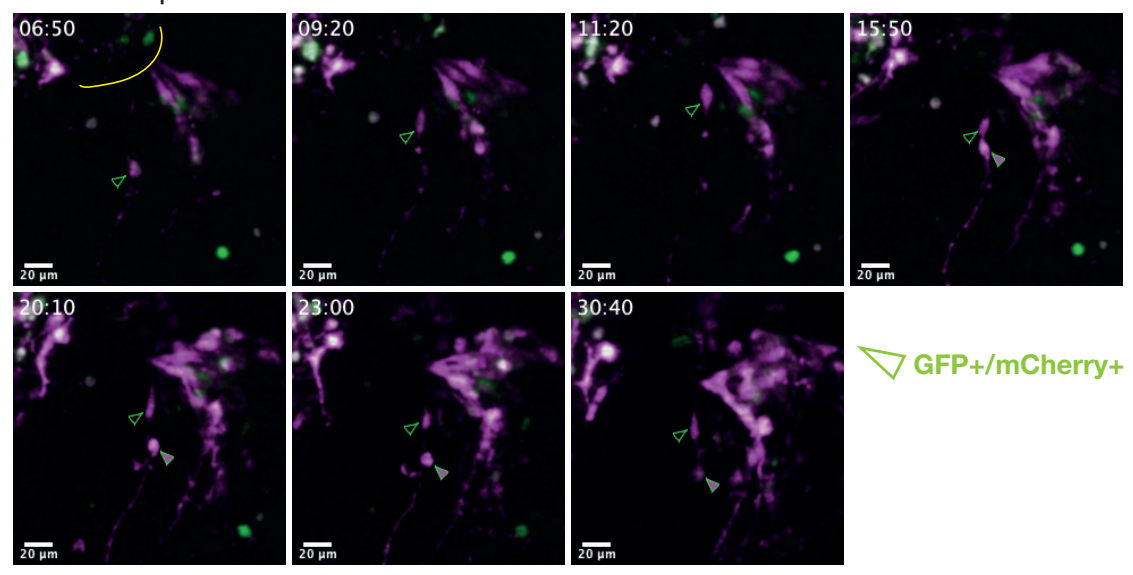

Extended Data Figure 4.

Still images from live imaging of organoids electroporated at a) $31 \mathrm{~d}$ and b) $54 \mathrm{~d}$, and imaged beginning at 2 days post-electroporation (see Methods for details of image acquisition). pCAG-mCherry+ cells are shown in magenta, and EGFP-C1-AR-V7+ are shown in green. Green arrowheads: GFP+ cells. Yellow arrowheads: mCherry+/GFP- cells. Filled in arrowheads: one of the tracked daughter cells. a) Comparison of the behaviour of mCherry+ and GFP+ only cells at 33d. Basal surface is up. mCherry+ only cell, upon division (06:11) produces two daughter cells, one of which migrates basally, indicating a more differentiated identity, while the other stays in the ventricular zone (VZ), representing an example of asymmetric division. GFP+ cell divides (07:28), but both of the daughter cells continue to reside in the VZ. b) Behaviour of an mCherry+/GFP+ cell at 56d. Basal surface

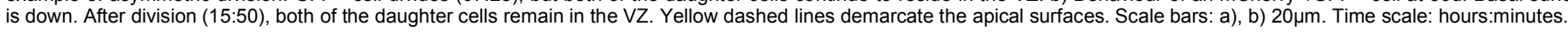




\section{Extended Data Figure 5.}

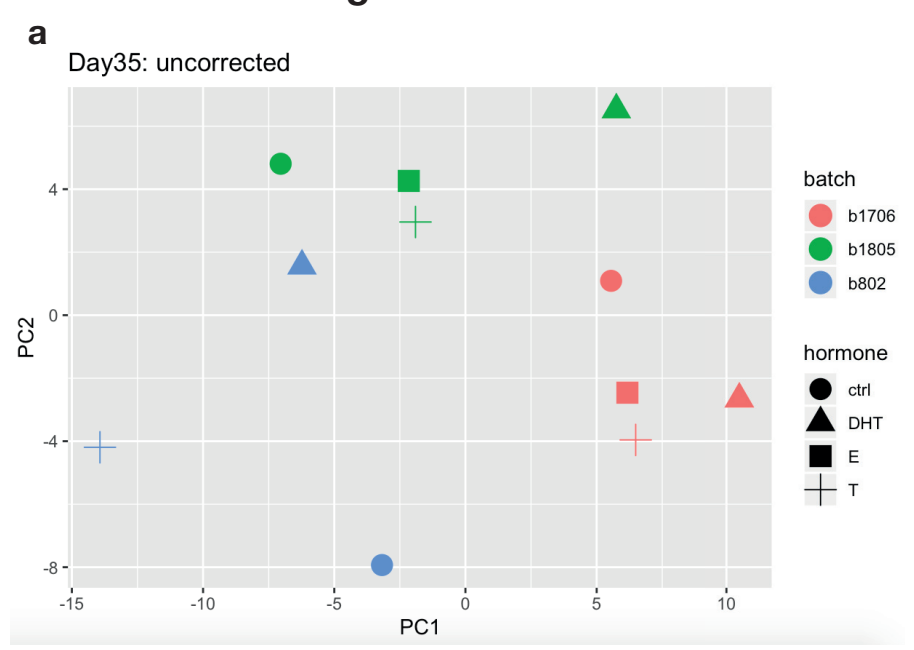

b
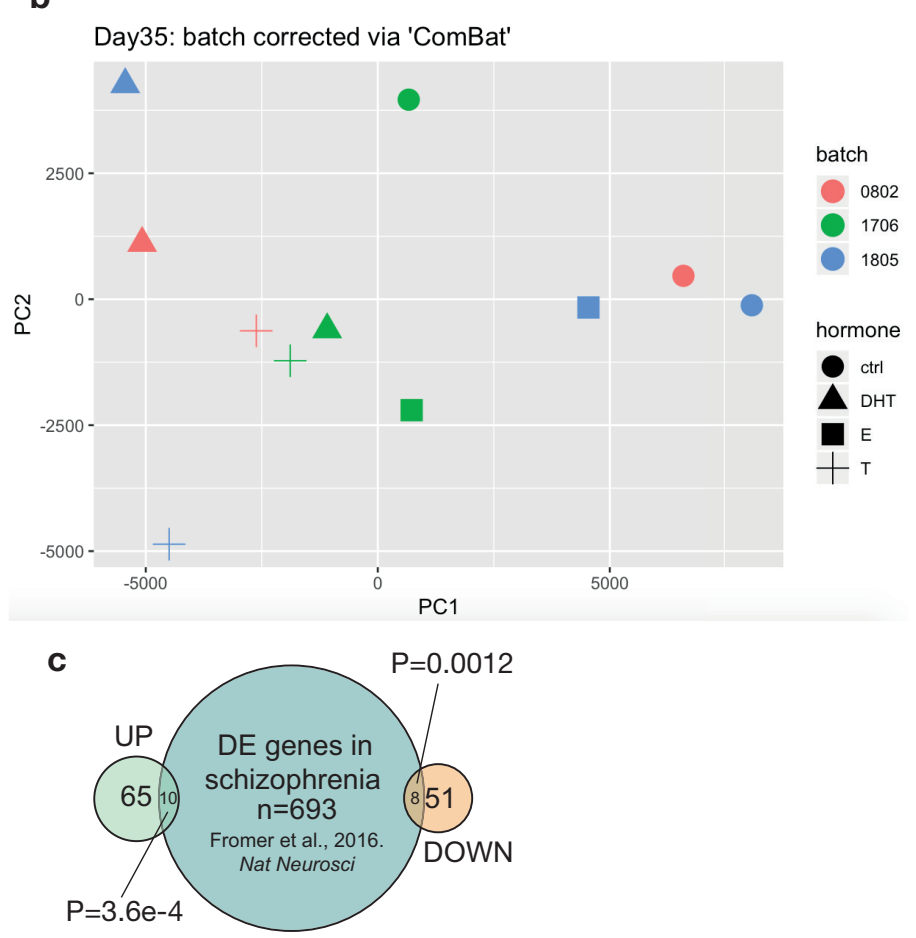

\section{Extended Data Figure 5.}

a), b) Principle component analysis (PCA) plots of XX 35d RNA-seq for both a) uncorrected and b) corrected for batch effects. c) Venn diagram representing the overlap of up- and downregulated differentially expressed genes (DEGs) in androgen treated XX 35d organoids with genes differentially expressed in schizophrenia patients ${ }^{42} . P$ values represent significance of the intersection between the datasets. DE: differentially expressed.

\section{Extended Data Movie 1.}

Live imaging of XX organoids, electroporated at $31 \mathrm{~d}$, and imaged at $33 \mathrm{~d}$ for $>48$ hours. Cells labelled with pCAG-mCherry are in magenta. Cells electroporated with EGFP-C1-AR-V7 are in green. Still images from this movie are shown in Extended Data Figure 4a. Time scale: hours:minutes.

\section{Extended Data Movie 2.}

Live imaging of XX organoids, electroporated at 54d, and imaged at 56d for $>48$ hours. Cells labelled with pCAG-mCherry are in magenta. Cells electroporated with EGFP-C1-AR-V7 are in green. Still images from this movie are shown in Extended Data Figure 4b. Time scale: hours:minutes.

\section{Extended Data Movie 3.}

Green channel (EGFP-C1-AR-V7) from Extended Data Movie 2. for easier tracking of electroporated, GFP+ cells. Time scale: hours:minutes. 
bioRxiv preprint doi: https://doi.org/10.1101/2020.10.24.353359; this version posted October 24, 2020. The copyright holder for this preprint (which was not certified by peer review) is the author/funder. All rights reserved. No reuse allowed without permission.

\begin{tabular}{|c|c|c|c|c|}
\hline \multicolumn{5}{|l|}{$\begin{array}{l}\text { UPREGULATED } \\
\text { DEGs }\end{array}$} \\
\hline ESEMBL ID & Gene name & Full name & $\begin{array}{l}\text { SFARI gene } \\
\text { (Gene score } \geq 3 \text { ) }\end{array}$ & DE in SZ (SZDB) \\
\hline ENSG00000065978 & YBX1 & Y-Box Binding Protein 1 & & \\
\hline ENSG00000066629 & EML1 & EMAP Like 1 & & \\
\hline ENSG00000070785 & EIF2B3 & Eukaryotic Translation Initiation Factor 2B Subunit Gamma & & \\
\hline ENSG00000082068 & WDR70 & WD Repeat Domain 70 & & \\
\hline ENSG00000089009 & RPL6 & Ribosomal Protein L6 & & \\
\hline ENSG00000100029 & PES1 & Pescadillo Ribosomal Biogenesis Factor 1 & & Yes \\
\hline ENSG00000100281 & HMGXB4 & HMG-Box Containing 4 & & Yes \\
\hline ENSG00000104738 & MCM4 & Minichromosome Maintenance Complex Component 4 & Yes & \\
\hline ENSG00000106628 & POLD2 & DNA Polymerase Delta 2, Accessory Subunit & & \\
\hline ENSG00000108179 & PPIF & Peptidylprolyl Isomerase F (Cyclophilin D) & & \\
\hline ENSG00000109919 & MTCH2 & Mitochondrial Carrier 2 & & Yes \\
\hline ENSG00000110080 & ST3GAL4 & ST3 Beta-Galactoside Alpha-2,3-SialyItransferase 4 & & \\
\hline ENSG00000116161 & CACYBP & Calcyclin Binding Protein & & \\
\hline ENSG00000116514 & RNF19B & Ring Finger Protein $19 \mathrm{~B}$ & & Yes \\
\hline ENSG00000121152 & NCAPH & Non-SMC Condensin I Complex Subunit H & & \\
\hline ENSG00000121390 & PSPC1 & Paraspeckle Component 1 & & \\
\hline ENSG00000121680 & PEX16 & Peroxisomal Biogenesis Factor 16 & & Yes \\
\hline ENSG00000123395 & ATG101 & Autophagy Related 101 & & \\
\hline ENSG00000123416 & TUBA1B & Tubulin Alpha $1 \mathrm{~b}$ & & \\
\hline ENSG00000124688 & MAD2L1BP & MAD2L1 Binding Protein & & \\
\hline ENSG00000129680 & MAP7D3 & MAP7 Domain Containing 3 & & \\
\hline ENSG00000130021 & PUDP & Pseudouridine 5'-Phosphatase & & \\
\hline ENSG00000132341 & RAN & RAN, Member RAS Oncogene Family & & \\
\hline ENSG00000134057 & CCNB1 & Cyclin B1 & & \\
\hline ENSG00000137691 & C11 orf70 (CFAP300) & Cilia And Flagella Associated Protein 300 & & \\
\hline ENSG00000137876 & RSL24D1 & Ribosomal L24 Domain Containing 1 & & \\
\hline ENSG00000138231 & DBR1 & Debranching RNA Lariats 1 & & \\
\hline ENSG00000138764 & CCNG2 & Cyclin G2 & & \\
\hline ENSG00000139921 & TMX1 & Thioredoxin Related Transmembrane Protein 1 & & \\
\hline ENSG00000143575 & HAX1 & HCLS1 Associated Protein X-1 & & \\
\hline ENSG00000145545 & SRD5A1 & Steroid 5 Alpha-Reductase 1 & & Yes \\
\hline ENSG00000148229 & POLE3 & DNA Polymerase Epsilon 3, Accessory Subunit & & Yes \\
\hline ENSG00000152465 & NMT2 & N-Myristoyltransferase 2 & & \\
\hline ENSG00000156508 & EEF1A1 & Eukaryotic Translation Elongation Factor 1 Alpha 1 & & \\
\hline ENSG00000162704 & ARPC5 & Actin Related Protein 2/3 Complex Subunit 5 & & \\
\hline ENSG00000164087 & POC1A & POC1 Centriolar Protein A & & \\
\hline ENSG00000164109 & MAD2L1 & Mitotic Arrest Deficient 2 Like 1 & & \\
\hline ENSG00000164758 & MED30 & Mediator Complex Subunit 30 & & \\
\hline ENSG00000165526 & RPUSD4 & RNA Pseudouridine Synthase D4 & & \\
\hline ENSG00000165775 & FUNDC2 & FUN14 Domain Containing 2 & & \\
\hline ENSG00000166347 & CYB5A & Cytochrome B5 Type A & & \\
\hline ENSG00000166557 & TMED3 & Transmembrane P24 Trafficking Protein 1 & & \\
\hline ENSG00000166851 & PLK1 & Polo Like Kinase 1 & & \\
\hline ENSG00000167085 & PHB & Prohibitin & Yes & \\
\hline ENSG00000167112 & TRUB2 & TruB Pseudouridine Synthase Family Member 2 & & \\
\hline ENSG00000168282 & MGAT2 & Alpha-1,6-Mannosyl-Glycoprotein 2-Beta-N-Acetylglucosaminyltransferase & & \\
\hline ENSG00000169714 & CNBP & CCHC-Type Zinc Finger Nucleic Acid Binding Protein & & \\
\hline ENSG00000170364 & SETMAR & SET Domain And Mariner Transposase Fusion Gene & & \\
\hline ENSG00000171720 & HDAC3 & Histone Deacetylase 3 & & \\
\hline ENSG00000172053 & QARS & Glutaminyl-TRNA Synthetase & & Yes \\
\hline ENSG00000176903 & PNMA1 & PNMA Family Member 1 & & \\
\hline ENSG00000180581 & SRPgP1 & Signal Recognition Particle 9 Pseudogene 1 & & \\
\hline ENSG00000182054 & IDH2 & Isocitrate Dehydrogenase (NADP(+)) 2 & & Yes \\
\hline ENSG00000183431 & SF3A3 & Splicing Factor 3a Subunit 3 & & \\
\hline ENSG00000183684 & ALYREF & Aly/REF Export Factor & & \\
\hline ENSG00000186665 & C17orf58 & Chromosome 17 Open Reading Frame 58 & & \\
\hline ENSG00000196262 & PPIA & Peptidylprolyl Isomerase A & & \\
\hline ENSG00000196591 & HDAC2 & Histone Deacetylase 2 & & Yes \\
\hline ENSG00000198039 & ZNF273 & Zinc Finger Protein 273 & & \\
\hline ENSG00000229119 & AC026403.1 & 60S acidic ribosomal protein (RPLPO) pseudogene & & \\
\hline ENSG00000244563 & RPS26P19 & Ribosomal protein S26 pseudogene 19 & & \\
\hline ENSG00000251668 & AC113404.2 & $\begin{array}{l}\text { pseudogene similar to part of microtubule associated serine/threonine } \\
\text { kinase } 2 \text { MAST2 }\end{array}$ & & \\
\hline ENSG00000255302 & EID1 & EP300 Interacting Inhibitor Of Differentiation 1 & & \\
\hline ENSG00000270800 & RPS10-NUDT3 & RPS10-NUDT3 readthrough & & \\
\hline ENSG00000280071 & GATD3B & Glutamine amidotransferase like class 1 domain containing $3 B$ & & \\
\hline
\end{tabular}


bioRxiv preprint doi: https://doi.org/10.1101/2020.10.24.353359; this version posted October 24, 2020. The copyright holder for this preprint (which was not certified by peer review) is the author/funder. All rights reserved. No reuse allowed without permission.

\begin{tabular}{|c|c|c|c|c|}
\hline $\begin{array}{l}\text { DOWNREGULATED } \\
\text { DEGs }\end{array}$ & & & & \\
\hline ESEMBL ID & Gene name & Full name & $\begin{array}{l}\text { SFARI gene } \\
\text { (Gene score } \geq 3 \text { ) }\end{array}$ & DE in SZ (SZDB) \\
\hline ENSG00000015568 & RGPD5 & RANBP2 Like And GRIP Domain Containing 5 & & Yes \\
\hline ENSG00000092929 & UNC13D & Unc-13 Homolog D & & \\
\hline ENSG00000099840 & IZUMO4 & IZUMO Family Member 4 & & \\
\hline ENSG00000100596 & SPTLC2 & Serine Palmitoyltransferase Long Chain Base Subunit 2 & & \\
\hline ENSG00000101104 & PABPC1L & Poly(A) Binding Protein Cytoplasmic 1 Like & & \\
\hline ENSG00000107317 & PTGDS & Prostaglandin D2 Synthase & & Yes \\
\hline ENSG00000108515 & ENO3 & Enolase 3 & & \\
\hline ENSG00000112855 & HARS2 & Histidyl-TRNA Synthetase 2, Mitochondrial & & \\
\hline ENSG00000116266 & STXBP3 & Syntaxin Binding Protein 3 & & \\
\hline ENSG00000123473 & STIL & STIL Centriolar Assembly Protein & & \\
\hline ENSG00000129003 & VPS13C (PARK23) & Vacuolar Protein Sorting 13 Homolog C & & \\
\hline ENSG00000132485 & ZRANB2 & Zinc Finger RANBP2-Type Containing 2 & & Yes \\
\hline ENSG00000136100 & VPS36 & Vacuolar Protein Sorting 36 Homolog & & \\
\hline ENSG00000137831 & UACA & Uveal Autoantigen With Coiled-Coil Domains And Ankyrin Repeats & & \\
\hline ENSG00000139219 & COL2A1 & Collagen Type II Alpha 1 Chain & & \\
\hline ENSG00000143416 & SELENBP1 & Selenium Binding Protein 1 & & Yes \\
\hline ENSG00000147654 & EBAG9 & Estrogen Receptor Binding Site Associated Antigen 9 & & \\
\hline ENSG00000148600 & CDHR1 & Cadherin Related Family Member 1 & & \\
\hline ENSG00000154222 & CC2D1B & Coiled-Coil And C2 Domain Containing 1B & & \\
\hline ENSG00000157036 & EXOG & Exo/Endonuclease $\mathrm{G}$ & & \\
\hline ENSG00000157379 & DHRS1 & Dehydrogenase/Reductase 1 & & \\
\hline ENSG00000160194 & NDUFV3 & NADH:Ubiquinone Oxidoreductase Subunit V3 & & Yes \\
\hline ENSG00000161298 & ZNF382 & Zinc Finger Protein 382 & & \\
\hline ENSG00000167264 & DUS2 & Dihydrouridine Synthase 2 & & \\
\hline ENSG00000168300 & PCMTD1 & $\begin{array}{l}\text { Protein-L-Isoaspartate (D-Aspartate) O-Methyltransferase Domain } \\
\text { Containing } 1\end{array}$ & & \\
\hline ENSG00000176809 & LRRC37A3 & Leucine Rich Repeat Containing 37 Member A3 & & \\
\hline ENSG00000179889 & PDXDC1 & Pyridoxal Dependent Decarboxylase Domain Containing 1 & & \\
\hline ENSG00000181085 & MAPK15 & Mitogen-Activated Protein Kinase 15 & & \\
\hline ENSG00000181666 & HKR1 (ZNF875) & Zinc Finger Protein 875 & & \\
\hline ENSG00000188234 & AGAP4 & ArfGAP With GTPase Domain, Ankyrin Repeat And PH Domain 4 & & \\
\hline ENSG00000196275 & GTF2IRD2 & GTF2I Repeat Domain Containing 2 & & \\
\hline ENSG00000196739 & COL27A1 & Collagen Type XXVII Alpha 1 Chain & & \\
\hline ENSG00000198804 & MT-CO1 & Mitochondrially Encoded Cytochrome C Oxidase I & & \\
\hline ENSG00000198840 & MT-ND3 & $\begin{array}{l}\text { Mitochondrially Encoded NADH:Ubiquinone Oxidoreductase Core Subunit } \\
3\end{array}$ & & \\
\hline ENSG00000198888 & MT-ND1 & $\begin{array}{l}\text { Mitochondrially Encoded NADH:Ubiquinone Oxidoreductase Core Subunit } \\
11\end{array}$ & & Yes \\
\hline ENSG00000204681 & GABBR1 & Gamma-Aminobutyric Acid Type B Receptor Subunit 1 & & \\
\hline ENSG00000205583 & STAG3L1 & Stromal Antigen 3-Like 1 (Pseudogene) & & \\
\hline ENSG00000225630 & MTND2P28 & MT-ND2 Pseudogene 28 & & \\
\hline ENSG00000226232 & NPIPB14P & Nuclear pore complex interacting protein family member B14, pseudogene & & \\
\hline ENSG00000228253 & MT-ATP8 & Mitochondrially encoded ATP synthase membrane subunit 8 & & \\
\hline ENSG00000232196 & MTRNR2L4 & MT-RNR2 Like 4 & & \\
\hline ENSG00000234441 & AC105272.1 & & & \\
\hline ENSG00000237732 & CT75 & Cancer/testis associated transcript 75 & & \\
\hline ENSG00000237973 & MTCO1P12 & MT-CO1 pseudogene 12 & & \\
\hline ENSG00000239912 & RPL39P36 & Ribosomal protein L39 pseudogene 36 & & \\
\hline ENSG00000243716 & NPIPB5 & Nuclear pore complex interacting protein family member B5 & & \\
\hline ENSG00000248527 & MTATP6P1 & MT-ATP6 pseudogene 1 & & \\
\hline ENSG00000256618 & MTRNR2L1 & MT-RNR2 Like 1 & & \\
\hline ENSG00000258366 & RTEL1 & Regulator Of Telomere Elongation Helicase 1 & & \\
\hline ENSG00000258890 & CEP95 & Centrosomal Protein 95 & & \\
\hline ENSG00000274615 & AC233968.1 & Aminopeptidase puromycin sensitive (NPEPPS) pseudogene & & \\
\hline
\end{tabular}

Extended Data Table 1. List of differentially expressed genes between control and androgen-treated organoids Up- and downregulated differentially expressed genes (DEGs) between control and androgen (DHT and T) -treated XX 35d organoids. 
bioRxiv preprint doi: https://doi.org/10.1101/2020.10.24.353359; this version posted October 24, 2020. The copyright holder for this preprint (which was not certified by peer review) is the author/funder. All rights reserved. No reuse allowed without permission.

Kelava et al. | bioRxiv | October 23, 2020 | 19 